\title{
Fusion Reactor Design Studies- Standard Unit Costs and Cost Scaling Rules
}

S. C. Schulte

W. E. Bickford

C. E. Willingham

S. K. Ghose

M. G. Walker

September 1979

Prepared for the U.S. Department of Energy under Contract EY-76-C-06-1830

Pacific Northwest Laboratory Operated for the U.S. Department of Energy by Battelle Memorial Institute 
NOTICE

This report was prepared as an account of work sponsored by the United States Government. Neither the United States nor the Department of Energy, nor any of their employees, nor any of their contractors, subcontractors, or their employees, makes any warranty, express or implied, or assumes any legal liability or responsibility for the accuracy, completeness or usefulness of any information, apparatus, product or process disclosed, or represents that its use would not infringe privately owned rights.

The views, opinions and conclusions contained in this report are those of the contractor and do not necessarily represent those of the United States Government or the United States Department of Energy.

\author{
PACIFIC NORTHWEST LABORATORY \\ operated by \\ BATTELLE \\ for the \\ UNITED STATES DEPARTMENT OF ENERGY \\ Under Contract EY-76-C-06-1830
}

\begin{tabular}{|c|c|}
\hline \multicolumn{2}{|c|}{$\begin{array}{l}\text { Printed in the United States of America } \\
\text { Available from } \\
\text { National Technical Information Service } \\
\text { Inited States Department of Commerce } \\
5285 \text { Port Royal Road } \\
\text { Springfield, Virginia } 22151\end{array}$} \\
\hline Printed Cop: & $\because$ Microfiche $\$ 3.00$ \\
\hline - Pages & $\begin{array}{c}\text { NTIS } \\
\text { Selling Price }\end{array}$ \\
\hline $001-025$ & $54: 00$ \\
\hline $026-050$ & $\$ 4.50$ \\
\hline $051-075$ & $\$ 5.25$ \\
\hline $076-100$ & $\$ 6.00$ \\
\hline $101-125$ & $\$ 6.50$ \\
\hline $126-150$ & $\$ 7.25$ \\
\hline $151-175$ & $\$ 8.00$ \\
\hline $176-200$ & $\$ 9.00$ \\
\hline $201-225$ & $\$ 9.25$ \\
\hline $226-250$ & $\$ 9.50$ \\
\hline $251-275$ & $\$ 10.75$ \\
\hline $276-300$ & $\$ 11.00$ \\
\hline
\end{tabular}


FUSION REACTOR DESIGN STUDIESSTANDARD UNIT COSTS AND COST SCALING RULES
S. C. Schulte
W. E. Bickford
C. E. Willingham
S. K. Ghose(a)
M. G. Walker(a)

September 1979

Prepared for

the U.S. Department of Energy under Contract EY-76-C-06-1830

Pacific Northwest Laboratory Richland, Washington 99352

(a) Bechtel National, Inc. San Francisco, California 94119 


\section{FOREWORD}

In preparation of this document, the DOE Office of Fusion Energy and the Pacific Northwest Laboratory arranged for the user community to review and agree to the content of the document. The utility of the document is based on this common satisfaction of the diverse needs and approaches of the user community.

To maintain this utility, this document should not be revised without first obtaining the concurrence of the Office of Fusion Energy that any proprosed revision will be compatible with the current needs and procedures of the magnetic fusion power development program. 
. 


\section{$\underline{\text { ABSTRACT }}$}

This report establishes standard unit costs and scaling rules for estimating costs of material, equipment, land, and labor components used in magnetic confinement fusion reactor plant construction and operation. Use of the standard unit costs and scaling rules will add uniformity to cost estimates, and thus allow valid comparison of the economic characteristics of various reactor concepts. 


\section{CONTENTS}

FOREWORD •

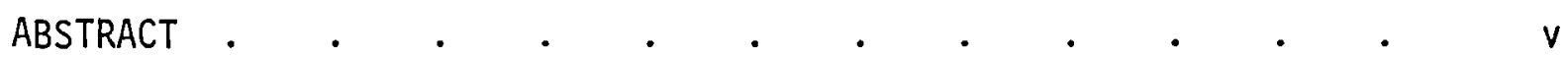

INTRODUCTION

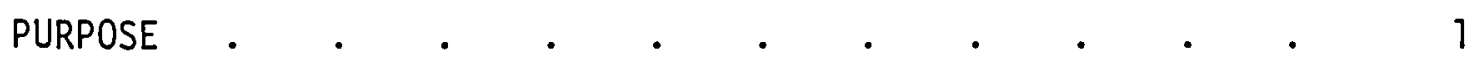

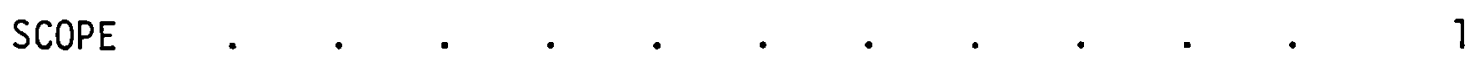

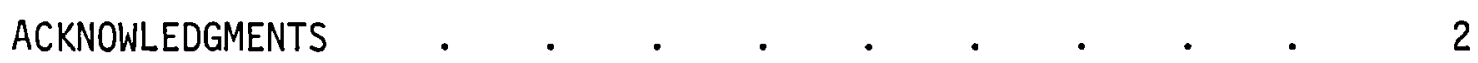

STANDARD UNIT COSTS AND COST ESTIMATING RULES..$\quad$. $\quad$. $\quad$. 3

ESCALATION METHODOLOGY

METHODOLOGY FOR ESTABLISHING CONSTRUCTION SCHEDULE. $\cdot \cdots$

BIBLIOGRAPHY

APPENDIX A - STANDARD UNIT COSTS . . . . . . . . . . A.1

APPENDIX B - REFERENCE POWER CONVERSION PLANT DESCRIPTION • • B. 1

APPENDIX C - REFERENCE POWER CONVERSION PLANT COST ESTIMATE • • C. 1

APPENDIX D - REFERENCE POWER CONVERSION PLANT COST ESTIMATE SCALING RULES $\quad \cdot \quad \cdot \quad \cdot \quad \cdot \quad \cdot \quad \cdot \quad \cdot \quad \cdot \quad$ D. 1

APPENDIX E - COST ESTIMATING RULES . • . • . • • • E.T

APPENDIX F - STANDARD UNIT COSTS . . . . . . . . . . F.1

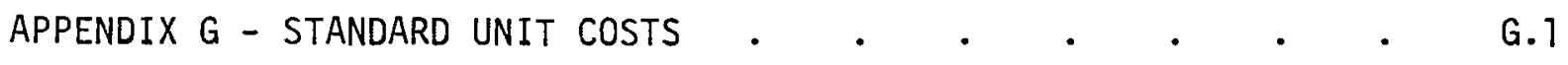


.

. 


\section{INTRODUCTION}

The Department of Energy and its contractors are engaged in the economic evaluation of fusion power reactor concepts. This document describes an economic evaluation format to be utilized in the evaluation of magnetic confinement fusion power reactor concepts. The format was developed by Battelle at the Pacific Northwest Laboratory for the Department of Energy. Use of the format will result in cost estimates that are generated on a uniform and consistent basis.

The evaluation format was developed in two phases. Phase I established standard cost estimate reporting accounts and a standard method for translating estimates of plant capital investment costs, operating and maintenance costs, scheduled component replacement costs, and fuel costs into an estimate of levelized energy cost. The reporting accounts and translating method are described in Fusion Reactor Design Studies - Standard Accounts for Cost Estimates, (PNL-2648, May 1978). Phase II established standard unit costs and cost scaling rules for estimating capital investment, operating, and fuel costs. These unit costs and scaling rules are described in this report.

PURPOSE

The evaluation format will provide the fusion community with a tool that will make cost estimates produced at different times and by different estimators comparable. This report defines uniform unit costs and scaling rules that will allow estimators to estimate costs based on identical assumptions and unit cost relationships. This report also defines a price level index that will allow cost estimates made in different years to be uniformly normalized to account for inflation. Using these rules, cost estimates can easily be made comparable.

$\underline{\text { SCOPE }}$

The cost estimating rules are applicable to commercial magnetic confinement fusion reactor power plant concepts constructed and operated in a mature fusion economy. The rules define standard unit costs and cost estimating relationships for estimating direct capital investment costs, engineering and 
administrative costs, and interest and escalation costs. Also defined are relationships for estimating operating and maintenance costs, scheduled component replacement costs, and fuel costs. The rules define a price level index for inflating the standard cost estimating relationships to reflect later year price levels. A reference plant construction schedule is also provided for use in those cases where the organization performing a reactor study does not have a better basis for estimating the length of construction.

\section{ACKNOWLEDGMENTS}

This report has been prepared by Battelle at the Pacific Northwest Laboratory (PNL) for the Office of Fusion Energy (OFE) of the United States Department of Energy under the guidance of Mr. Charles Head.

The authors thank the following people for their contributions:

D. Sonn, General Atomic Company

G. Carlson, Lawrence Livermore Laboratory

W. Neef, Lawrence Livermore Laboratory

D. DeFreece, McDonne11-Douglas Astronautics Company

L. Waganer, McDonne11-Douglas Astronautics Company

L. Carose11a, McDonne11-Douglas Astronautics Company

L. Reid, Oak Ridge National Laboratory

J. Kelly, Westinghouse Electric Corporation 


\section{STANDARD UNIT COSTS AND COST ESTIMATING RULES}

The contents of this report consist of standard unit costs, cost scaling rules and reference plant subsystem cost estimates. Unit costs are installed costs per unit quantity $(\mathrm{e} . \mathrm{g} ., \$ / \mathrm{kg}, \$ / 1 \mathrm{~b})$. Reference plant subsystem cost estimates are generic balance of plant subsystem cost estimates.

The unit costs and cost scaling rules are established for a cost estimating level of detail commensurate with the level of detail currently achieved in fusion reactor cost estimating. If too many unit costs and scaling rules are established, application of the data by different estimators may result in nonuniform costing of identical subsystems. If too few unit costs and scaling rules are established, the cost estimating process may not be flexible enough to permit the identification of significant cost differences.

This report is intended to be used for estimating installed costs for commercial fusion power reactor components constructed and operated in a mature fusion economy. The unit costs and cost scaling rules are based on July 1979 price levels. However, costing data for use in subsequent years can be generated by applying the process outlined on Page 5 of this report.

The standard unit costs and cost scaling rules are arranged as follows:

Cost Account
Land and Land Rights (Account 20)
Site Improvements and Facilities (Account 21.01)
Reactor Building (Account 21.02)

Turbine Building (Account 21.03)

Cooling System Structures (Account 21.04)
Standard unit costs for land and land

Index
Standard unit costs for land and land
rights are listed in Appendix A.
Standard unit costs for site improvements
and facilities, and reactor building are
listed in Appendix A.
A cost estimate for building and cooling
structures associated with a reference
1,000 Me turbine plant is listed in
Appendix C. Cost estimating rules for
scaling this cost estimate to power con-
version systems in the range from 800 to
1,400 MWe are given in Appendix $D$.
rights are listed in Appendix $A$.
Standard unit costs for site improvements and facilities, and reactor building are
A cost estimate for building and cooling structures associated with a reference 1,000 Me turbine plant is listed in Appendix C. Cost estimating rules for version systems in the range from 800 to 1,400 MWe are given in Appendix $D$.


Power Supply and Energy Storage Buildings (Account 21.05)

Miscellaneous Buildings (Account 21.06) Ventilation Stacks (Account 21.07)

Reactor Plant Equipment (Áccount 22)

Turbine Plant Equipment (Account 23)

Electric Plant Equipment (Account 24)

Miscellaneous Plant Equipment (Account 25)

Special Materials (Account 26)

Construction Facilities, Equipment, and Services (Account 91)

Engineering and Construction Management Services (Account 92)

Other Costs (Account 93)

Interest During Construction (Account 94)

Escalation During Construction (Account 95)

Spare Parts Allowance

Design Al lowance

Contingency Al lowance

Operating and Maintenance (Account 40-47)

Scheduled Component Replacement (Account 50-51)

Fuel (Account 02-03)
Standard unit costs for power supply and energy storage buildings, miscellaneous buildings and ventilation stacks are listed in Appendix A.

Standard unit costs for reactor plant equipment are listed in Appendix $A$.

$A$ cost estimate for turbine piant equipment associated with a reference 1,000 MWe turbine plant is listed in Appendix $C$. Cost estimating rules for scaling this cost estimate to power conversion systems in the range from 800 to 1,400 MWe are given in Appendix $D$.

A cost estimate for electric plant equipment associated with a reference 1,000 MWe turbine plant is listed in Appendix $C$. Cost estimating rules for scaling this cost estimate to power conversion systems in the range from 800 to 1,400 MWe are given in Appendix $D$.

A cost estimate for miscellaneous plant equipment associated with a reference 1,000 We turbine is 1 isted in Appendix $C$. Cost estimating rules for scaling this cost estimate to power conversion systems in the range from 800 to 1,400 MWe are given in Appendix 0 .

Standard unit costs for special materials are listed in Appendix G.

Cost estimating rules for construction facilities, equipment and services, and for engineering and construction management services and other costs are given in PNL-2648.

Cost estimating rules for interest and escalation during construction are given in PNL-2648.

Cost estimating rules for spare parts, design and contingency allowances are listed in Appendix E.

Standard unit costs for operating and maintenance and scheduled component replacement are 1 isted in Appendix F. Gross cost estimating rules are given in PNL-2648.

Standard unit costs for fuel are 1 isted in Append ix $G$. 


\section{ESCALATION METHODOLOGY}

Fusion reactor component costs will change over time in response to general price level change (i.e., inflation), changes in market conditions (i.e., supply and demand), and technological changes. As a result, the standard unit costs and cost estimating rules will require periodic updating. The escalation methodology defined in this report will provide estimators with a tool to uniformly update the standard cost estimating rules. The updating mechanism will allow 1) cost estimates to be based on current year price levels (and not just mid-1979 price levels) and 2) estimates developed in different years to be put on a same year price level basis and, therefore, made comparable.

The escalation methodology utilizes price level index inflation factors. A single index is designated for use with each unit cost or cost estimating rule. Cost estimates developed during calendar year 1979 should utilize the standard unit costs and cost scaling rules as they are stated in this report. Cost estimates developed in later years should utilize escalated costs (i.e., costs that have been updated via the designated inflation factors to reflect price levels for the year in which the cost estimate is performed). The year for which the cost estimate is made should be stated in the cost estimate.

The inflation factors (price level indexes) are contained in the following publications:

- The Handy Whitman Index of Public Utility Construction Costs. Whitman, Requardt, and Associates, Baltimore, MD. (Electric Utility Construction for the North Central Region.)

- Survey of Current Business. United States Department of Commerce, Bureau of Economic Analysis, Washington, DC. (General Business Indicators, national income and product implicit price deflators, gross national product.)

The sources of inflation factors (price level indexes) to be used with each unit cost or cost estimating rule are shown in Table 1 . A11 unit costs and cost estimating rules reported under the same major reporting account utilize the same index. 


\section{TABLE 1. Sources of Price Level Indexes (Inflation Factors)}

Cost Account

Land and Land Rights (Account 20)

Structures and Site Facilities (Account 21)

Reactor Plant Equipment (Account 22)

Turbine Plant Equipment (Account 23)

Electric Plant Equipment (Account 24)

Miscellaneous Plant Equipment (Account 25)

Special Materials (Account 26)

Construction Facilities, Equipment, and Services (Account 91)

Engineering and Construction Management Services (Account 92

Other Costs (Account 93)

Interest During Construction (Account94)

Escalation During Construction - Optional (Account 95)

Spare Parts Allowance

Design Allowance

Contingency Allowance

Operating and Maintenance (Account 40-47)

Scheduled Component Replacement (Account 50-51)

Fuel (Account 02-03)
Index

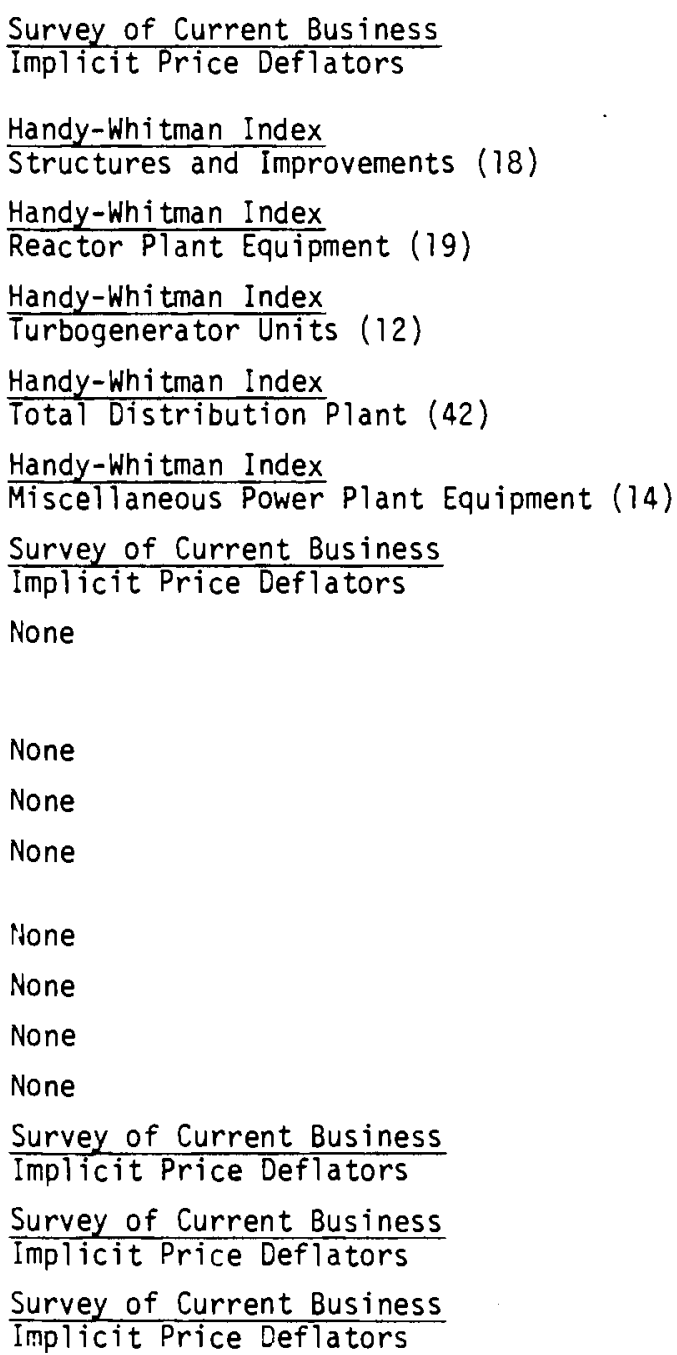

Application of the inflation factors will only approximate cost data in future years. Over time, major reporting account inflation factors will not adequately reflect changes in unit costs and cost estimating rules. For this reason, price level index inflation factors should not be utilized after fiscal year 1982 .

An example of the application of a price level index inflation factor is given below: 


\section{EXAMPLE}

Objective

Determine Turbine Generator and Accessories Cost (23.01.01) for a July 1981 cost estimate

Given

Turbine Generator and Accessories Cost

$(1979$ dollars $)=\$ 64.5 \mathrm{million}$

Appendix C

Inflation Factor Index = Handy Whitman

Turbogenerator Units

Table 1

Handy Whitman Cost Index Number - July

$1981=460$

Hypothetical

Calculations

Current year cost $=$

$\frac{\text { inflation factor index (current year) }}{\text { inflation factor index (Ju?y 1979) }} \times \operatorname{cost~(JuTy~1979)~}$

July 1981 Turbine Generator and Accessories

Cost $=$

$\frac{460}{388} \times \$ 64.5$ million $=\$ 76.5$ million 


\section{METHODOLOGY FOR ESTABLISHING CONSTRUCTION SCHEDULE}

The purpose of this section is to provide guidelines for estimating fusion reactor plant construction schedules. A reference fusion plant construction schedule is established for use in studies in which there is no specific basis for establishing construction schedule. It should be understood that these guidelines serve to provide approximations in the absence of detailed data on design, engineering, procurement, and construction. Power plant construction schedules are generally considered to cover the entire time period during which capital expenditures are made in direct support of a power plant project. Thus, the schedule includes project-specific preliminary design studies, construction permit and licensing activities, detailed design and engineering, procurement, construction, fuel loading, testing, and startup.

The importance of properly estimating schedule durations is apparent from inspection of the magnitude of the time-related elements of capital costs. The combination of escalation during construction and interest during construction through an extended construction schedule can account for more than half the entire capital cost of a large power plant project.

For the purpose of estimating schedule requirements for a fusion reactor power plant, an 11 year length of construction should be utilized. Due to institutional and market uncertainties and the early state of fusion reactor designs, a typical Light water Reactor (LWR) construction schedule was assumed to approximate fusion reactor plant construction schedule. The reference schedule is shown in Figure 1. 


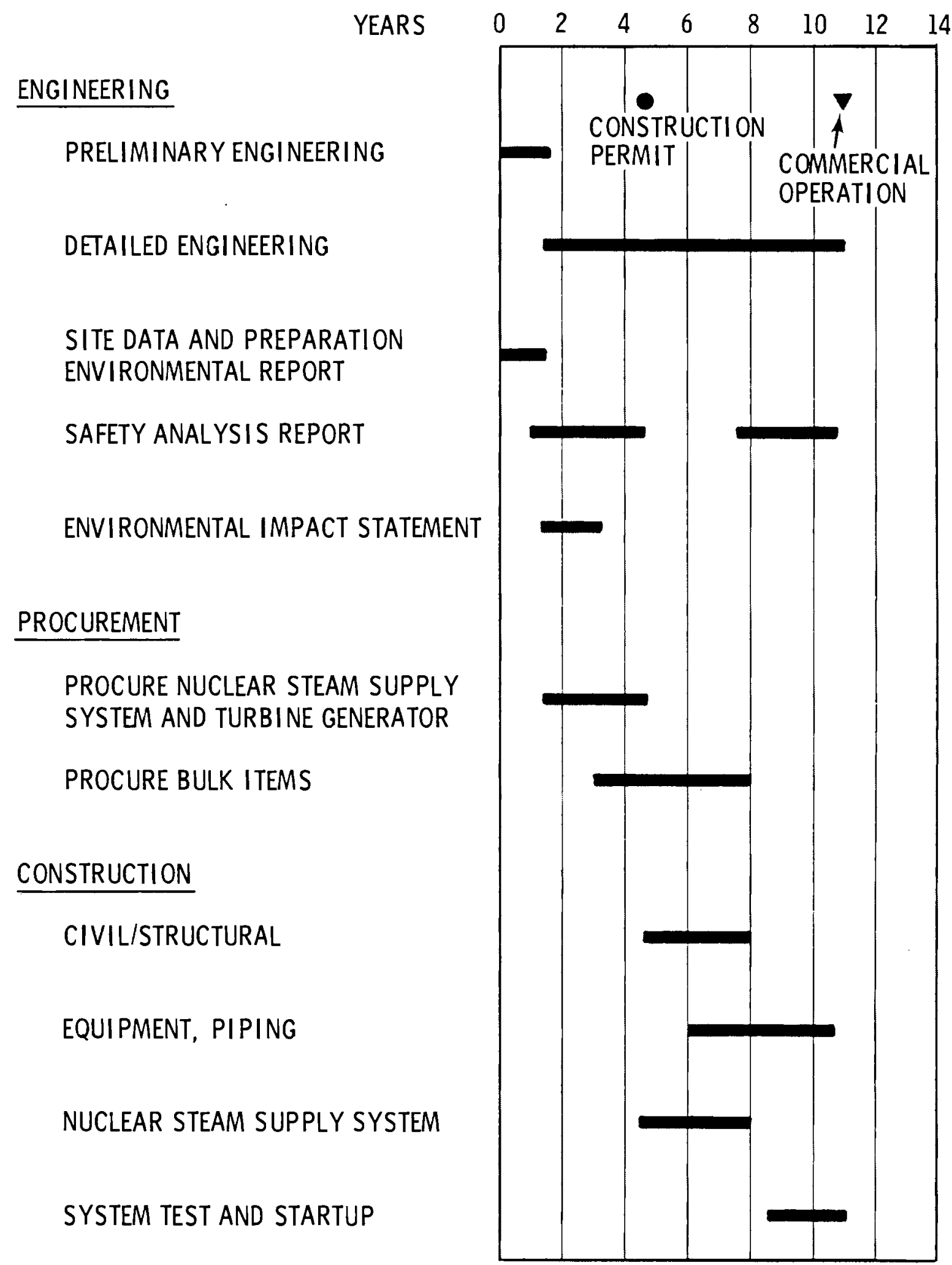

FIGURE 1. Reference Schedule 
Badger, B., et a1. June 1977. Tokamak Engineering Test Reactor. Nuclear Engineering Department, University of Wisconsin, Madison, Wisconson.

Carlson, G. A., et al. November 15, 1978. Status Report on Tandem Mirror Fusion Reactor Studies. Lawrence Livermore Laboratory, University of Cal ifornia, Livermore, California.

Chapin, D. L. and Gibson, G. September 30, 1977. TNS Engineering Trade Study Analysis. Fusion Power Systems Department, Westinghouse Electric Corporation, Pittsburg, Pennsylvania.

Chen, w., et a1. July 1978. Experimental Fusion Power Reactor Conceptual Design Study, Vol. I, I I, I I. General Atomic Report to National Technical Information Service, U.S. Dept. of Commerce, Springfield, Virginia.

Coultas, T. A., et al. 1976. Cost Estimation for a Theta Pinch Reactor. Engineering Division, Argonne National Laboratory, Argonne, Illinois.

Fusion Engineering Staff. November 1976. Conceptual Study of a Noncircular Tokamak Demonstration Fusion Power Reactor. General Atomic Company.

Garber, H. J. and Sniderman, M. April 14, 1977. Fusion Power Systems Technical Note Costing Models for TNS Tokamaks. Westinghouse Electric Corporation, Fusion Power Systems Department, Pittsburg, Pennsylvania.

Hoffman, M. A. January 23, 1978. Direct Energy Converters for Fusion Reactors/ Efficiency and Cost for Two Electrostatic Concepts. Lawrence Livermore Laboratory, University of California, Livermore, California.

Hovingh, J. August 1977. First Wa11 Costs of an Ion Beam Fusion Reactor. Lawrence Livermore Laboratory, University of California, Livermore, California.

Howard, H. R., et a1. May 1977. Design Study of a Fusion-Driven Tokamak Hybrid Reactor for Fissile Fuel Production Final Report, Vol. I and II. Westinghouse Electric Corporation, Fusion Power Systems Department, Pittsburg, Pennsyivania.

Materials Engineering. Mid-September, 1975. 1976 Materials Selector. Vol. 84, No. 4.

McDonne11-Douglas Astronautics Company. October 1976. Fusion Reactor First Wa11/Blanket Systems Analys is Final Report. St. Louis, Missouri.

McDonne11-Douglas Astronautics Company. October 1977. Fusion Reactor First Wa 11/Blanket Systems Analys is Tokamak Concepts - Research Report (interim). St. Louis, Missouri. 
Moir, R. W., et a1. June 25, 1975a. Progress on the Conceptual Design of a Mirror Hybrid Fusion-Fission Reactor. Lawrence Livermore Laboratory, University of California, Livermore, California.

Moir, R. W., et a1. June 25, 1975b. Standard Mirror Fusion Reactor Design Study. Lawrence Livermore Laboratory, University of California, Livermore, California.

Moir, R. W., et al. January 30, 1978a. Preliminary Design of the Tandem Mirror Reactor. Lawrence Livermore Laboratory, University of California, Livermore, California.

Moir, R. W., et a1. January 30, 1978b. Standard Mirror Fusion Reactor Design Study. Lawrence Livermore Laboratory, University of California, Livermore, California.

Powe11, J. R. February 25, 1972. Costs of Magnets for Large Fusion Power Reactors: Phase I, Cost of Superconductors for DC Magnets. Department of Applied Science, Brookhaven National Laboratories, Associated Universities, Inc., Upton, New York for the Division of Thermonuclear Research, United States Atomic Energy Commission, Washington, DC.

Scanlan, R. M. October 14, 1975. Memo: Superconducting Materials for Future Magnets: Cost Projections. Lawrence Livermore Laboratory, University of California, Livermore, California.

Sommer, D. W. January 22, 1979. "Statistics Sti11 Showing." Economic Trends/ Industry Week. (Abstract)

Steiner, D. March 1977. ORNL Fusion Power Demonstration Study: Interim Report. Union Carbide Corporation, Oak Ridge National Laboratory, Oak Ridge, Tennessee.

TNS Engineering Staff. January 1977. TNS Quarterly Progress Report for the Period October, November December $(1976)$. Fusion Power Systems Department, Westinghouse Electric Corporation, Pittsburg, Pennsylvania.

Varljen, T. L., Gibons, G., French, J. W. and Heck, F. M. September 30, 1977. Engineering Parameters for Four Ignition Reactor Systems. Fusion Power Systems Department, Westinghouse Electric Corporation, Pittsburg, Pennsylvania. 


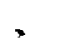


APPENDIX A

STANDARD UNIT COSTS

Land/Structures and Site Facilities/

Reactor Plant Equipment 
STANDARD UNIT COSTS - Land, Structures and Equipment

"Installed cost consists of material and equipnient purchase expense, fabrication/assembly expense, transportation/purchasing/testing expense, direct and indirect installation expense, and associated overhead expense."

A. 1 
Installed Cost

(7/79 Price Levels)

20. LAND AND LAND RIGHTS

Land and Land Rights

$\$ 2500 /$ Acre

21. STRUCTURES AND SITE FACILITIES

Excavation

Fill and Compaction

Concrete Work (in place)

Reinforcing Steel
$\$ 3.40 / \mathrm{cu} \mathrm{yd}$

$\$ 3.60 / \mathrm{cu}$ yd

$\$ 230 /$ cu yd

$\$ 300 / \mathrm{cu}$ yd

22. REACTOR PLANT EQUIPMENT

22.01 Reactor Equipment

22.01.01 Blanket and First Wall

Wall Modifiers (coating, liners, etc.)

Carbon/graphite (woven cloth,

u100 $\mathrm{um}$ thick)

$\$ 220 / \mathrm{kg}$

Carbon/graphite (plain liner

thin squares, $27 \mathrm{~cm}$ thick)

$\$ 25 / \mathrm{kg}$

Pyrolitic graphite $(.5 \mathrm{~cm}$ thick

liner)

$\$ 3000 / \mathrm{kg}$

SiC ( $1 \mathrm{~cm}$ thick, radiating panels)

$\$ 265 / \mathrm{kg}$

Tungsten ( $p$ lates sintered

from powder)

$\$ 80 / \mathrm{kg}$

$\mathrm{Al}_{2} \mathrm{O}_{3}$ ( $1 \mathrm{~cm}$ thick 1 iner $)$

$\$ 25 / \mathrm{kg}$

Graphite (honeycomb structure)

$\$ 300 / \mathrm{kg}$

First Wall

Tubular (thin wal1, $2.5 \mathrm{~cm} \mathrm{00,}$ $0.06 \mathrm{~cm}$ wall)

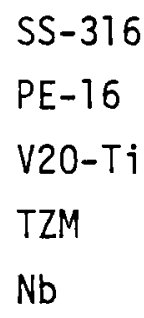

Other
$\$ 14 / \mathrm{kg}$

$\$ 20 / \mathrm{kg}$

$\$ 184 / \mathrm{kg}$

$\$ 170 / \mathrm{kg}$

$\$ 88 / \mathrm{kg}$ 
First Wa11 (contd)

Sheet (segmented torus, no support)

\begin{tabular}{|c|c|}
\hline$S S-316$ & $\$ 25 / \mathrm{kg}$ \\
\hline V20-Ti & $\$ 200 / \mathrm{kg}$ \\
\hline $\mathrm{Nb}$ & $\$ 150 / \mathrm{kg}$ \\
\hline TZM & $\$ 190 / \mathrm{kg}$ \\
\hline Inconel-718 & $\$ 49 / \mathrm{kg}$ \\
\hline \multicolumn{2}{|l|}{ other } \\
\hline \multicolumn{2}{|c|}{$\begin{array}{l}\text { lodular or Bucket Shells } \\
\text { complicated fabrication, thin wall, } \\
\text { igid quality control and testing) }\end{array}$} \\
\hline SS-316 & $\$ 32 / \mathrm{kg}$ \\
\hline V20-Ti & $\$ 250 / k$ \\
\hline Incone1-718 & $\$ 60 / \mathrm{kg}$ \\
\hline TZM & $\$ 240$ \\
\hline
\end{tabular}

Blanket

Highly machined components, forgings, castings (lattices, seats, locking rings)

SS-316

Incone -718

Blanket structure (fabricated from heavy plates/beams by welding, cutting, bolting)

SS-316

Inconel-718

$\mathrm{Nb}$

TZM

Other

Breeding Material (assume reactor grade 1ithium)

Liquid Lithium (natural ${ }^{6} L i$ )
Liquid Lithium (90\% enriched
$6 L i$ )
$\$ 25 / \mathrm{kg}$

$\$ 49 / \mathrm{kg}$

$\$ 15 / \mathrm{kg}$

$\$ 26 / \mathrm{kg}$

$\$ 90 / \mathrm{kg}$

$\$ 169 / \mathrm{kg}$

$\$ 30 / \mathrm{kg}$

$\$ 1000 / \mathrm{kg}$ 
Breeding Material (contd)

Sol id Li Compounds (natural

in SS cans, $10-25 \mathrm{~cm} \mathrm{OD)}$

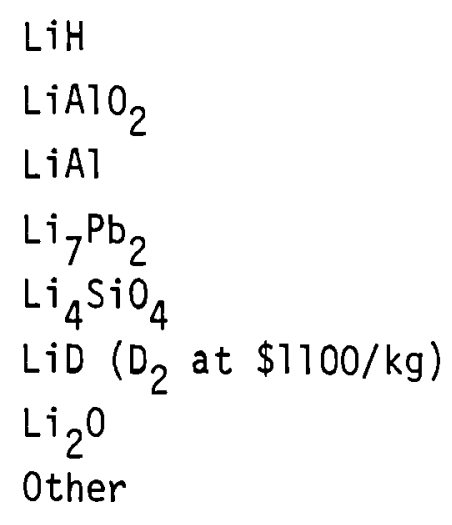

$\$ 35 / \mathrm{kg}$

$\$ 40 / \mathrm{kg}$

$\$ 40 / \mathrm{kg}$

$\$ 45 / \mathrm{kg}$

$\$ \cdot 8 / \mathrm{kg}$

$\$ 250 / \mathrm{kg}$

$\$ 33 / \mathrm{kg}$

Solid Li Compounds (natural, in SS pins or rods $\leq 3 \mathrm{~cm} \mathrm{OD)}$

LiH

$\mathrm{LiAlO}_{2}$

LiA1

$\mathrm{Li}_{7} \mathrm{~Pb}_{2}$

$\mathrm{Li}_{4} \mathrm{SiO}_{4}$

LiD

$\mathrm{Li}_{2} \mathrm{O}$

Other

Solid Li Compounds (90\% enriched

$\sigma_{L i}$, in $S S$ cans, $10-25 \mathrm{~cm} \mathrm{OD)}$

$\mathrm{LiAlO}_{2}$
$\mathrm{Li}_{4} \mathrm{SiO}_{4}$
$\mathrm{LiAl}$
$\mathrm{LiD}$
$\mathrm{Li}_{2} \mathrm{O}$

Other

Solid Li Compounds (90\% enriched

$6 L i$, clad in SS pins or rods

$\leq 3 \mathrm{~cm} \mathrm{OD)}$
$\mathrm{LiAlO}_{2}$
$\mathrm{Li}_{4} \mathrm{SiO}_{4}$

$\$ 52 / \mathrm{kg}$

$\$ 60 / \mathrm{kg}$

$\$ 60 / \mathrm{kg}$

$\$ 68 / \mathrm{kg}$

$\$ 12 / \mathrm{kg}$

$\$ 375 / \mathrm{kg}$

$\$ 50 / \mathrm{kg}$
$\$ 150 / \mathrm{kg}$

$\$ 54 / \mathrm{kg}$

$\$ 160 / \mathrm{kg}$

$\$ 1020 / \mathrm{kg}$

$\$ 500 / \mathrm{kg}$

$\$ 234 / \mathrm{kg}$

$\$ 81 / \mathrm{kg}$ 
Installed cost

(7/79 Price Levels)

Breeding Material (contd)

LiA1

$\$ 234 / \mathrm{kg}$

LiD

$\$ 1500 / \mathrm{kg}$

$\mathrm{Li}_{2} \mathrm{O}$

$\$ 750 / \mathrm{kg}$

Other

Attenuators, Reflectors and Multi-

pliers (in SS cans or bulk quantities)

$\mathrm{C}$

$\mathrm{Be}$

$\$ 6 / \mathrm{kg}$

$\$ 220 / \mathrm{kg}$

$\mathrm{Pb}$

$\$ 3 / \mathrm{kg}$

Other

Attenuators, Reflectors and Multipliers (clad in SS pins or rods $\leq 3 \mathrm{~cm} \mathrm{OD)}$

C

$\mathrm{Be}$

$\$ 9 / \mathrm{kg}$

$\mathrm{Pb}$

$\$ 330 / \mathrm{kg}$

$\$ 5 / \mathrm{kg}$

Other

22.01.02 Shield (primary and secondary)

C/graphite (blocks)

$\$ 6 / \mathrm{kg}$

$\mathrm{B}_{4} \mathrm{C}$ (in cans or bulk quantities)

$\$ 56 / \mathrm{kg}$

$\mathrm{Pb}$ (s'neets)

$\$ 3 / \mathrm{kg}$

SS-316 (sheets)

$\$ 15 / \mathrm{kg}$

SS-316 (pe11ets)

$\$ 10 / \mathrm{kg}$

Concrete

$\$ 265 / \mathrm{kg}^{3}$

Lead Cement

$\$ 2 / \mathrm{kg}$

Borated Water

$\$ 15 / \mathrm{kg}$

22.01.03 Magnets

Stabilizer

OFHC Cu (plain ribbon)

$\$ 5 / \mathrm{kg}$

OFHC Cu (stamped for cooling)

$\$ 10 / \mathrm{kg}$ 
Stabilizer (contd)

OFHC Cu (complex machining)

$\$ 25 / \mathrm{kg}$

A1

$\$ 11 / \mathrm{kg}$

Other

Superconductor

NbTi (copper matrix 1:1)

$\$ 50 / \mathrm{kg}$

$\mathrm{Nb}_{3} \mathrm{Sn}\left(\mathrm{Nb}_{3} \mathrm{Sn}\right.$ to bronze ratio, $1: 3$ )

$\$ 100 / \mathrm{kg}$

other

Conductor Bonding to Stabilizer

Soldering, Brazing (one surface)

$\$ 3 / \mathrm{kg}$

Wrap around with soldering

(multiple surfaces)

$\$ 6 / \mathrm{kg}$

Insulators

Micarta

$\$ 4 / \mathrm{kg}$

Epoxy

Super Thermal Insulation

$\$ 12 / \mathrm{kg}$

other

Internal Conductor Support

SS-316 (D shaped or circular discs with machined grooves

for conductor)

$\$ 25 / \mathrm{kg}$

SS-316 (spool or bobbin for solenoid type coil)

$\$ 20 / \mathrm{kg}$

SS-316 (Yin-Yang)

$\$ 30 / \mathrm{kg}$

Fabrication (includes fixtures, winding and assembly)

$$
\begin{array}{ll}
\text { Yin-Yang in shop } & \$ 50 / \mathrm{m} \\
\text { Toroidal, D or circular in shop } & \$ 45 / \mathrm{m} \\
\text { Toroidal or circular in field } & \$ 60 / \mathrm{m}
\end{array}
$$

Liquid He Dewar, see 22.03.01, Magnet Cooling

Dewar Vacuum Tank, see 22.01.06, Reactor Vacuum System 
22.01.04 Supplemental Heating (includes

power supply equipment)

RF Heating (based on output power)

Electron cyclotron resonance $\$ 5 / \mathrm{W}$

Lower hybrid resonance heating $\$ 1 / W$

Ion cyclotron resonance heating

$\$ .50 / \mathrm{W}$

Neutral Beam (based on output power)

$\$ 1.50 / W$

22.01.05 Primary Structure and Support

External Coil Supports

SS-316 Toroidal, Circular,

Distributed Structure

$\$ 20 / \mathrm{kg}$

Blanket and Shield Support

Simple Structure SS-316

Machined or fabricated SS-316

$\$ 20 / \mathrm{kg}$

Hardware 316-SS

$\$ 25 / \mathrm{kg}$

PCRV (prestressed, reinforced concrete)

$\$ 16 / \mathrm{kg}$

other

Direct Converter Vacuum Tank Support

SS-316 Support

$\$ 12 / \mathrm{kg}$

Concrete Support

$\$ 260 /$ cu yd

22.01.06 Reactor Vacuum System

Plasma Chamber Vacuum

Main Pumping System (includes

valving and piping)

Cryopumps $\left(\sim 10^{5}\right.$ liter $/ \mathrm{sec} / \mathrm{m}^{2}$

pump speed down to $10^{-8}$ torr)

Turbomolecular (up to $5 \times 10^{3}$

$\$ 0.50 / \mathrm{kg}$

liters/sec, backup for cryopumps)

$\$ 3000 / \mathrm{m}^{2}$

$\$ 150,000 /$ unit 
Reactor Vacuum System (contd)

Backup Pumps

Roots blower

(2500-3000 liter/sec)

Other

Roughing Pumps ( 100 liters/sec)

Supplemental Heating

Cryopumps $\left(10^{5}\right.$ liter $/ \mathrm{sec} / \mathrm{m}^{2}$

speed, pulsed, high gas loads)

Direct Converter Vacuum

Vacuum Tank (SS-316)

Pumps (cryopumps)

Vacuum Exhaust Ducts

$200 \mathrm{~cm}$ diameter SS-316

$100 \mathrm{~cm}$ diameter SS-316

$\sim 75 \mathrm{~cm}$ diameter $\mathrm{SS}-316$
$\$ 12,000 /$ unit

$\$ 6,000 /$ unit

$\$ 3,000 / \mathrm{m}^{2}$

$\$ 13 / \mathrm{kg}$

$\$ 3,000 / \mathrm{m}^{2}$

$\$ 20,000 / \mathrm{m}$

$\$ 15,000 / \mathrm{m}$

$\$ 10,000 / \mathrm{m}$

22.01.07 Power Supply Switching and Energy

Storage

Supplemental Heating (see 22.01.04)

Pulsed Coil Applications:

Flywheels (with AC-DC generator motor set)

$\$ 500 / \mathrm{MJ}$

Capacitor

$\$ 350,000 / \mathrm{MJ}$

D.C. Homopolar

$\$ 16,000 / \mathrm{MJ}$

Steady-State Coil Applications:

D.C. Solid State

$\$ 40 / \mathrm{kW}$

22.01.08 Impurity Control

Wa11 Trea tment

Low Z 1iners (see 22.01.01, Wal1 modifiers)

Honeycomb (see 22.01.01, Wal1 modifiers)

A. 8 
Installed Cost

(7/79 Price Levels)

Impurity Control (contd)

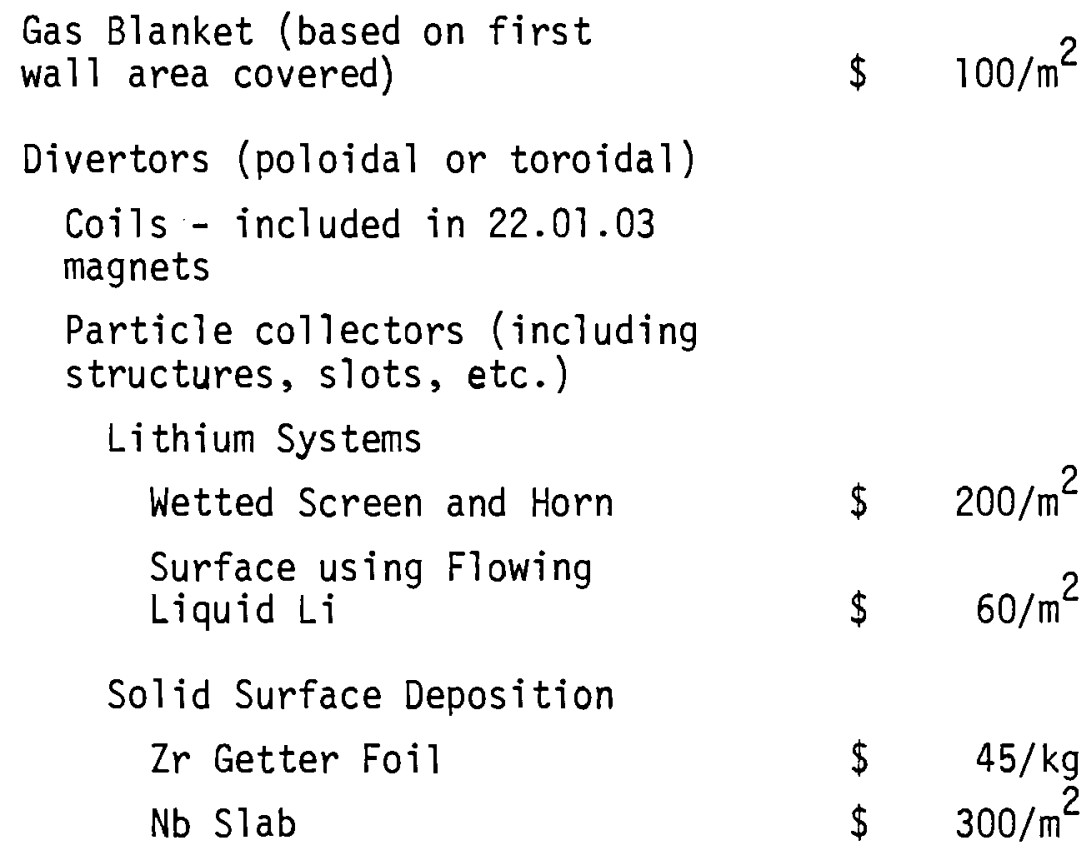

22.01.09 Direct Converter

Vacuum Tank SS-316, $1 \mathrm{~cm}$ thick $\$ 13 / \mathrm{kg}$

D.C. Modules

single-stage

Two-stage

Three-stage

Thermal Shields

Cryopumps ( $\mathrm{i}$ iquid He)

$\begin{array}{ll}\$ & 4,000 / \mathrm{m}^{2} \\ \$ & 7,000 / \mathrm{m}^{2} \\ \$ & 4,000 / \mathrm{m}^{2} \\ \$ & 3,500 / \mathrm{m}^{2} \\ \$ & 3,000 / \mathrm{m}^{2}\end{array}$

Liquid Nitrogen Refrigerators (based on power needed to operate liquefier)

$\$ 40,000 \times(k W)^{\cdot 7}$

Liquid Hel ium Refrigerators (based on power needed to operate i iquefier)

$\$ 40,000 \times(k W)^{.7}$

Power Conditioning (output from D.C. modules)

$\$ 44 / \mathrm{kW}$ 
22.02 Main Heat Transfer

\subsubsection{Primary Coolant System}

Pumps and Motor Drives

He Circulators $\left(25 \times 10^{4} \mathrm{~kg} / \mathrm{hr}\right.$ at 800 psi)

Liquid Metal $\left(\sim 2 \times 10^{6} \mathrm{~kg} / \mathrm{hr}\right.$ at 40 psi)

Liquid Metal $\left(\sim 1 \times 10^{7} \mathrm{~kg} / \mathrm{hr}\right.$ at 40 psi)

Piping (includes insulation)

Stainless Steel for He, 800900 psi

150-200 cm diameter

$<150 \mathrm{~cm}$ diameter

Stainless Steel for Liquid Metal

$50 \mathrm{~cm}$ diameter

Heat Exchangers

$$
\begin{aligned}
& \mathrm{Li} \rightarrow \mathrm{Na} \\
& \mathrm{He} \rightarrow \mathrm{Na}
\end{aligned}
$$

Tanks (SS-316, $400 \mathrm{~m}^{3}$ capacity)

Dump, Make-up or Hot Storage

$$
\text { Water }
$$

$\mathrm{Na}$

Li
$\$ 3,500,000 /$ unit

$\$ 1,000,000 /$ unit

$\$ 2,000,000 /$ unit

$$
\begin{array}{lr}
\$ & 100,000 / \mathrm{m} \\
\$ & 80,000 / \mathrm{m}
\end{array}
$$

$\$ 150,000 / \mathrm{m}$

Cleanup System

Circulating He (based on mass of coolant)

$\$ 2 / \mathrm{kg}$

Circulating Liquid Metals

$$
\begin{array}{ll}
\mathrm{Na} \text { (based on mass of coolant) } & \$ 5 / \mathrm{kg} \\
\mathrm{Li} \text { (based on mass of coolant) } & \$ 12 / \mathrm{kg}
\end{array}
$$


Installed Cost

(7/79 Price Levels)

22.02.02 Intermediate Coolant System

Pumps and Motor Drives

$$
\text { Water } \begin{aligned}
\left(\sim 10^{5} \mathrm{~kg} / \mathrm{hr}\right) & \$ 175,000 / \text { unit } \\
\left(\sim 10^{4} \mathrm{~kg} / \mathrm{hr}\right) & \$ 25,000 / \text { unit }
\end{aligned}
$$

Liquid Metal

$$
\begin{aligned}
& 210^{5} \mathrm{~kg} / \mathrm{hr} \\
& 210^{4} \mathrm{~kg} / \mathrm{hr}
\end{aligned}
$$

$\$ 300,000 /$ unit

$\$ 40,000 /$ unit

Piping (includes insulation)

$$
\begin{array}{ll}
\text { Water (SS-316, } 50 \mathrm{~cm} \text { diameter) } & \$ 8,000 / \mathrm{m} \\
\begin{array}{l}
\text { Water (carbon steel, } 50 \mathrm{~cm} \\
\text { diameter) }
\end{array} & \$ 3,500 / \mathrm{m} \\
\text { Liquid Metal (<50 cm diameter) } & \$ 16,000 / \mathrm{m}
\end{array}
$$

Heat Exchangers (steam generators)

$$
\begin{aligned}
& \mathrm{He} \rightarrow \text { Steam } \\
& \mathrm{Li} \rightarrow \text { Steam } \\
& \mathrm{Na} \rightarrow \text { Steam } \\
& \text { Water } \rightarrow \text { Steam }
\end{aligned}
$$

Tanks (SS-316, $400 \mathrm{~m}^{3}$ capacity)

Dump, Make-up or Hot Storage

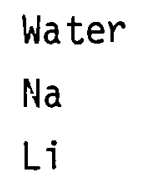

$\$ 115 / \mathrm{m}^{3}$

$\$ 950 / \mathrm{m}^{3}$

$\$ 1100 / \mathrm{m}^{3}$

Cleanup System (based on mass of coolant)

$$
\begin{aligned}
& \text { He System } \\
& \mathrm{Na} \text { System } \\
& \text { Li System }
\end{aligned}
$$
$\$ 2 / \mathrm{kg}$
$\$ 5 / \mathrm{kg}$
$\$ 12 / \mathrm{kg}$

\subsection{Auxiliary Cooling System}

$$
\text { 22.03.01 Magnet Cooling }
$$


Magnet Cooling (contd)

Superconducting Coil Dewar:

(SS-316 double wal1 vacuum

and coolant tank)

$$
\begin{aligned}
& \text { Yin-Yang, SS-316 } \\
& \text { D or Circular Shape }
\end{aligned}
$$

$\$ 30 / \mathrm{kg}$

$\$ 35 / \mathrm{kg}$

Liquid He Storage Dewar

$\$ 3.00 / 1$ iter

Liquid $\mathrm{N}_{2}$ Storage Dewar

$\$ 2.50 / 1$ iter

Refrigeration

$3 \mathrm{~kW}, 4.2 \mathrm{~K} \mathrm{He}$ Refrigerator

$\$ 2,000,000 /$ unit

Piping

$$
5 \mathrm{~cm} \text { OD SS-316 Schedule } 40
$$

$\$ 120 / \mathrm{m}$

22.03.02 Shield and Structure Cooling

Cooling (based on $\mathrm{kW}$ deposited)

$$
\begin{aligned}
& \text { Water } \\
& \mathrm{He}
\end{aligned}
$$

Piping (SS-316 Schedule 40)

$$
\begin{aligned}
& 5 \mathrm{~cm} \text { OD } \\
& 2 \mathrm{~cm} \text { OD }
\end{aligned}
$$

Pumps and Drives

$$
\begin{aligned}
& \text { Water }\left(\sim 10^{5} \mathrm{~kg} / \mathrm{hr}\right) \\
& \mathrm{He}\left(\sim 10^{4} \mathrm{~kg} / \mathrm{hr}\right)
\end{aligned}
$$

Heat Exchanger

$$
\mathrm{He} \rightarrow \text { Water }
$$

Purification

22.03.03 Supplemental Heating; Cooling

Injector Cooling (per $\mathrm{kW}$ deposited)

Piping (2 cm 0D, SS-316 Schedule 40)
$\$ 4 / \mathrm{kW}_{\text {th }}$
$\$ 6 / \mathrm{kW}_{\text {th }}$

$\$ 120 / m$

$\$ 100 / m$

$\$ 7 / \mathrm{kW}_{\text {th }}$ $\$ 25 / \mathrm{kW}_{\text {th }}$

$\$ 10 / \mathrm{kW}_{\text {th }}$

$\$ 1.5 / \mathrm{kW}_{\text {th }}$

$\$ 20 / \mathrm{kW}_{\text {th }}$

$\$ 100 / \mathrm{m}$ 
Supplemental Heating; Cooling (contd)

$$
\begin{aligned}
& \text { Pumps (water, } 210^{4} \mathrm{~kg} / \mathrm{hr} \text { ) } \\
& \text { Purification }
\end{aligned}
$$

22.03.04 Power Supply Cooling

Pumps, Piping, Cooling

22.04 Radioactive Waste Treatment and Disposal

(based on reactor thermal output)

Liquid Waste Processing (concrete
and ultimate disposal of tritiated
water

Gaseous Waste and Off-Gas Processing Systems

Solid Wastes Processing Equipment

22.05 Fuel Handling and Storage Systems

Fuel Purification (based on daily hydrogen mass flow rate)

Fuel Preparation and Injection System

D-T Pellet Fabrication (based on daily flow)

Gas Liquefiers

Fuel Storage

$20 \mathrm{~kg} \mathrm{~T}_{2}$ capacity

$20 \mathrm{~kg} \mathrm{D} \mathrm{D}_{2}$ capacity

Fuel Preparation (20 kg/day)

Fuel Injectors $(20 \mathrm{~kg} /$ day $)$

Emergency Air Detritiation

Facility Atmosphere Cleanup

(based on flow rate in the equipment)
Installed Cost

(7/79 Price Levels)

$\$ 2 / \mathrm{kW}_{\text {th }}$

$\$ 2.50 / \mathrm{kW}_{\text {th }}$

$\$ 3 / k W_{\text {th }}$

$\$ 400 / \mathrm{MW}_{t}$

$\$ 300 / \mathrm{MW}_{t}$

$\$ 1,000 / \mathrm{kg}-$ day

$\$ 12,000 / \mathrm{kg}-$ day

$\$ 3,800 / \mathrm{kg}$-day

$\$ 80,000 / \tan k$

$\$ 60,000 /$ machine

$\$ 250,000 /$ injector

$\$ 30 / \mathrm{gram} / \mathrm{T}$

$\$ 400 / \mathrm{cfm}$ 
• 


\section{APPENDIX B}

REFERENCE POWER CONVERSION PLANT DESCRIPTION 


\section{REFERENCE POWER CONVERSION PLANT DESCRIPTION}

1000 MWe GROSS

[For qualifications regarding this appendix, refer to Appendix C.]

\section{B.1 INTRODUCTION}

Reference 1000 MWe gross power conversion plants are described in this appendix. The reference plants are assumed to represent typical power conversion plants that can be integrated with magnetically confined fusion reactors and their associated heat transport systems.

The conceptual designs developed to date for this class of fusion reactors and their heat transport systems cover a wide spectrum of plant capacity, reactor heat source, coolant type, and coolant temperature. Examples of typical variation of these parameters are shown below:

\begin{tabular}{ll}
\multicolumn{1}{c}{ Parameter } & \multicolumn{1}{c}{ Range of Variation } \\
$\begin{array}{l}\text { Plant Capacity } \\
\text { Heat Source }\end{array}$ & $\begin{array}{l}\text { Blanket, Divertor, Direct } \\
\text { Convertor, etc. }\end{array}$ \\
Coolant Type & $\begin{array}{l}\text { Lithium, Helium, Water, } \\
\text { etc. }\end{array}$ \\
$\begin{array}{l}\text { Coolant Temperature } \\
\text { (reactor outlet) }\end{array}$ & $440^{\circ} \mathrm{C}$ to $980^{\circ} \mathrm{C}$
\end{tabular}

These wide variations have resulted in wide variations in the heat transport systems and power conversion systems in the conceptual fusion plant designs. The power conversion systems adopted in these designs range from state-of-the-art low temperature, saturated steam cycles to advanced systems like the closed cycle gas turbine (Brayton) cycle and the potassium cycle. In these advanced systems, attempts were made to utilize the high temperature available from fusion reactors to achieve high thermodynamic efficiencies. But, considering the adverse economics, lowered reactor first wall life, reliability and plant availability, technology development requirements, etc., 
imposed by high coolant temperatures, the current design trend has been to use lower temperatures. Low coolant temperatures have favorable impacts on these considerations.

Considering the trend toward lower temperatures, the reactor coolants are categorized into two groups for the purpose of standardizing power conversion systems for fusion conceptual design studies. These two groups are gas coolants (helium, steam, etc.) and liquid coolants (1ithium, water, molten salt, etc). The primary motivation for categorizing the coolants in this manner is to take advantage of the power conversion technologies already developed or being developed for the light water reactor (LWR), liquid metal fast breeder reactor (LMFBR), and gas-cooled fission reactor systems.

Representative heat transport systems with the associated power conversion systems for the two groups of coolants are shown in Figures $B-1$ and $B-2$. The saturated steam cycle has been largely used with moderate temperature liquid coolants, whereas the superheat steam cycle has been used mostly with high temperature gas coolants. The saturated cycle has been used in the LWR and LMFBR designs, while the superheat cycle has been used in LMFBR and gas-cooled reactor designs. Thus, for the fusion plants, two reference power conversion cycles are assumed -- a saturated steam cycle and a superheat steam cycle. The descriptions of the two cycles and the conversion plant arrangements are given in the following sections.

\section{B.2 SATURATED CYCLE}

A schematic diagram of the saturated cycle conversion system is shown in Figure $B-1$. The steam cycle selected for energy conversion is similar to that for LWR and LMFBR designs. Steam conditions at the turbine inlet are $6.9 \mathrm{MPa}$ and $285^{\circ} \mathrm{C}\left(1000 \mathrm{psia}\right.$ and $\left.545^{\circ} \mathrm{F}\right)$.

The turbine-generator selected is a tandem compound 1800 rpm unit operating with a saturated cycle. The unit consists of one high pressure (HP) cylinder, four moisture separator-reheaters, two low pressure (LP) cylinders, and one generator. 


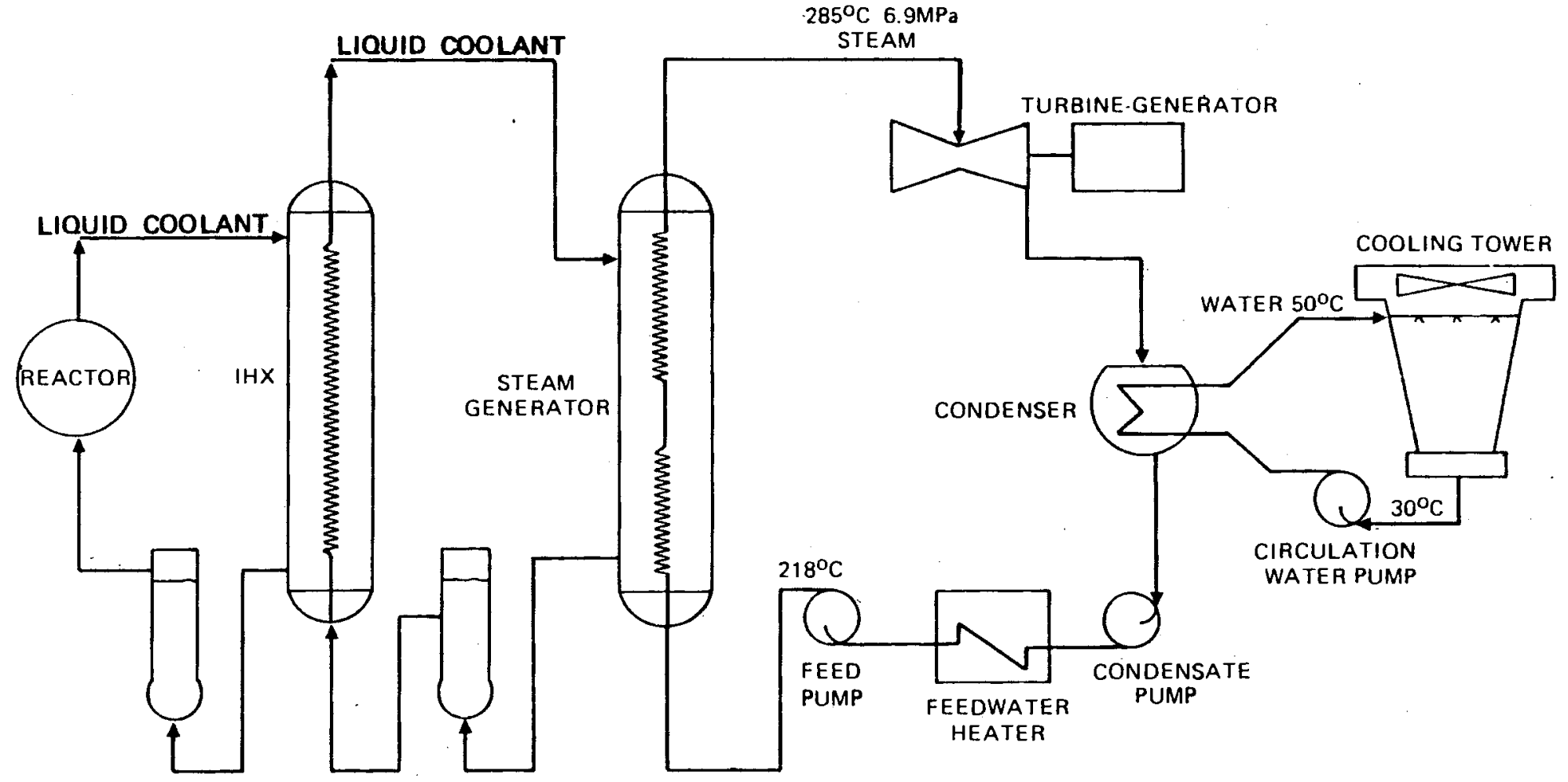

FIGURE B-1. Typical Heat Transport and Energy Conversion Systems for Liquid-Cooled Concepts 


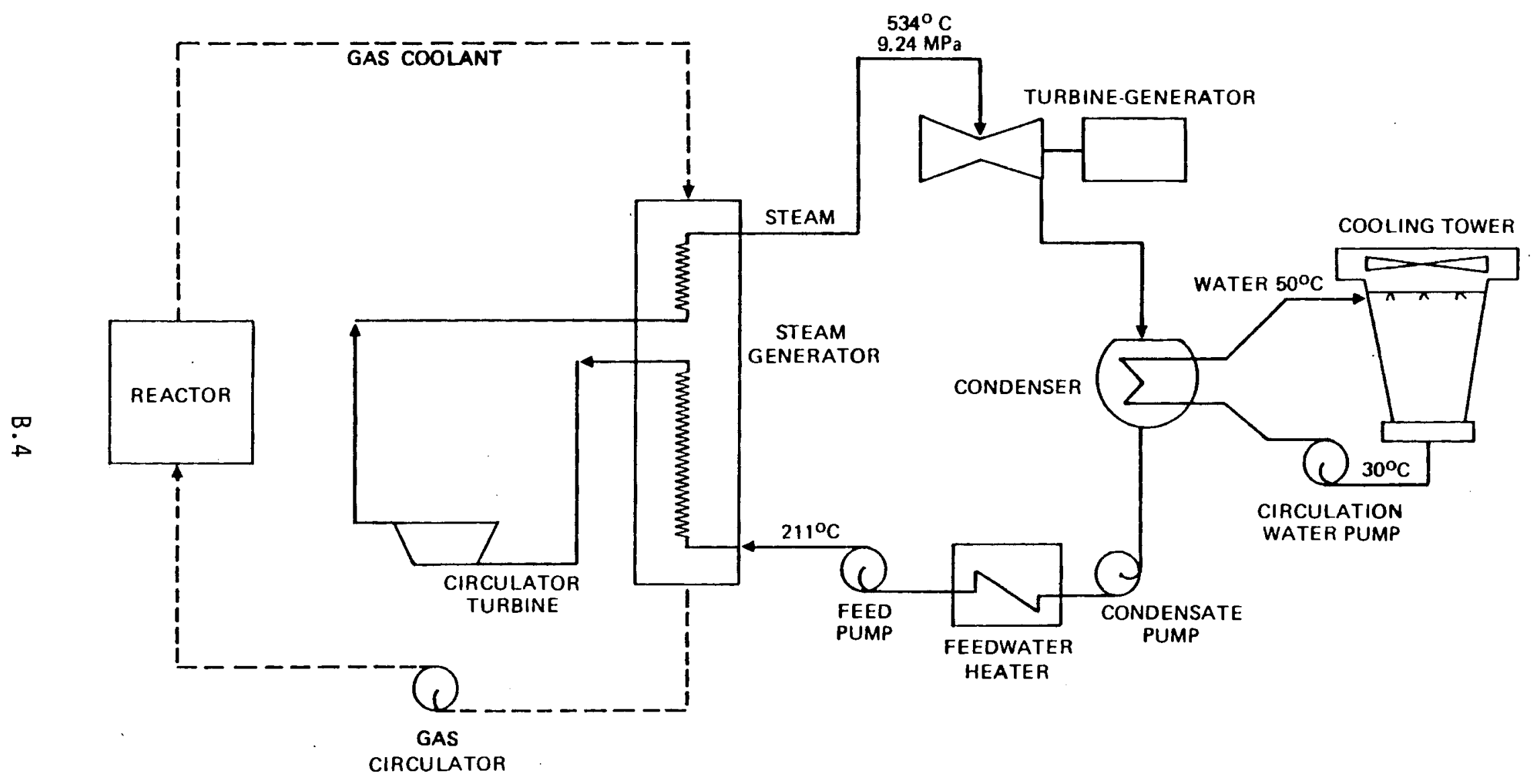

HEAT TRANSPORT SYSTEM

ENERGY CONVERSION SYSTEM HEAT REJECTION SYSTEM

FIGURE B-2. Typical Heat Transport and Energy Conversion Systems for Gas-Cooled Concepts 
The conversion system also includes a condensate system and a feedwater heating system. The function of the condensate system is to condense the steam exhausted from the low pressure cylinders of the turbine-generator unit. The condensate system schematicaliy consists of a steam condenser and a condensate pump which delivers the condensate to the feedwater heating system. The condensation is accomplished by circulating cold water through the condenser.

The function of the feedwater heating system is to preheat and pressurize the condensate and deliver it to the steam generator at the required temperature and pressure conditions. The system schematically consists of a feedwater pump(s) and feedwater heater(s). The pump is driven by a steam turbine. The heating of feedwater is accomplished by extracting steam from the turbine at different stages and passing it through feedwater heaters.

A large amount of heat is rejected from the energy conversion system while condensing the turbine exhaust steam in the steam condenser. This rejected heat amounts to approximately $65 \%$ of the total heat generated in the reactor. This is caused by the thermodynamic inefficiency of the Rankine cycle used in the conversion system.

The function of the heat rejection system is to reject this waste heat to an ultimate heat sink. This function is accomplished by transporting the heat rejected in the condenser to cooling towers where it is ultimately rejected to the atmosphere. The system schematically consists of a circulating water pump and a cooling tower as shown in Figure B-1. The circulating water pump draws cold water from the cooling tower and delivers it to the condenser to cool the condensing steam. The effluent hot water from the condenser then flows back to the cooling tower and exchanges heat with air.

A schematic heat and mass balance diagram of the conversion system is shown in Figure B-3. 


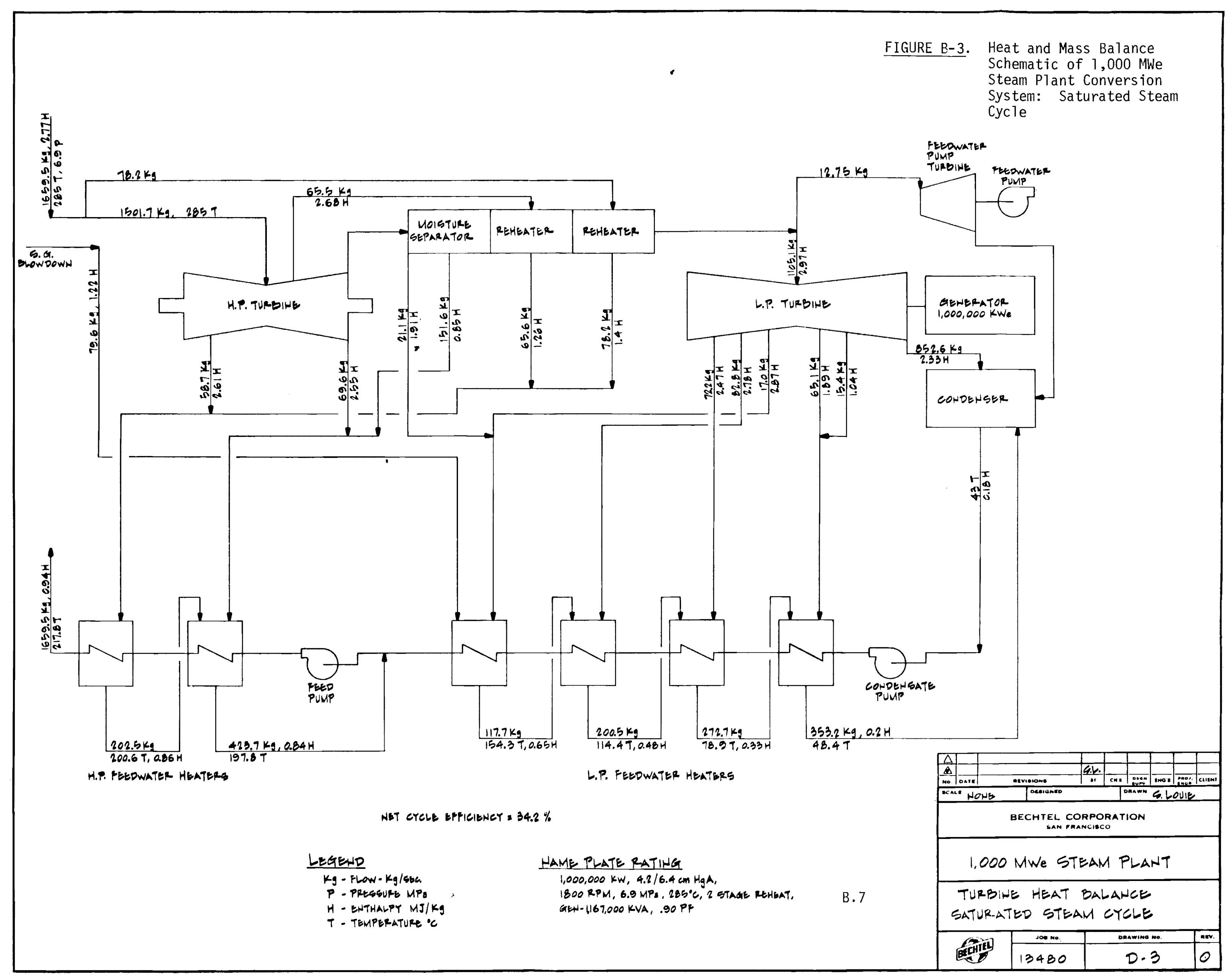


Major equipment along with their capacity ratings for the saturated cycle are listed below:

Main Steam Turbine

Type

Rating, kW

Speed, rpm

Inlet conditions, $\mathrm{MPa} /{ }^{\circ} \mathrm{C}$

Outlet conditions, cm $\mathrm{HgA}$

Steam flow, $\mathrm{kg} / \mathrm{sec}$
TC-4F-43 LSB

$1,000,000$

1,800

$6.9 / 285$

$6.35 / 4.19$

1501.7

Generator

Type

Rating, kVA/kV

Hydrogen pressure, $\mathrm{MPa}$

Power factor

4 pole, 3 phase, $60 \mathrm{~Hz}$

$1,167,000 / 25$

0.62

0.9

Feedpump Turbine

Type

Number

Rating, kW

Steam flow, $\mathrm{kg} / \mathrm{sec}$

Inlet conditions, $\mathrm{MPa} / \mathrm{MJ} / \mathrm{kg}$

Moisture Separator Reheater

Type

Number

Turbine Condensers

Type

Duty, MWt

Heat transfer surface, $\mathrm{m}^{2}$

Tube material

Cooling water flow, $\mathrm{kg} / \mathrm{sec}$

Circulating Water Pumps

Type

Number required

Capacity, $\mathrm{kg} / \mathrm{sec}$
Multistage, variable speed

2

8,400

12.75

$1.45 / 2.97$

Two-stage steam reheat, she11, and tube 4

Two she11, dual pressure, surface condenser

1,900

69,700

$90-10 \mathrm{Cu} \mathrm{Ni}$

37,500

Vertical-centrifugal wet-pit

4

8,000 


\section{EQUIPMENT LIST (contd)}

Circulating Water Pumps (contd)

Rated head, m

Motor rating, $\mathrm{kW}$

Cooling Tower

Type

Number of rows

Number of cells/row

Design bases, ambient $W B,{ }^{\circ} \mathrm{C}$ rated load

Approach, ${ }^{\circ} \mathrm{C} /$ range, ${ }^{\circ} \mathrm{C}$

Total rating, MWt

Total flow, $\mathrm{kg} / \mathrm{sec}$

Feedwater Heater

Type

Number, HP/LP

Final feedwater temperature Material

Condensate Pumps

Type

Number

Capacity, kg/sec

Head, m

Feedwater Pumps

Type

Number

Capacity, kg/sec

Head, m

Driver
32

3,400

Mechanical draft, wet cooling towers

3

14

15.6

$8.3 / 14.4$

1,960

31,500

Closed horizontal shell and tube 2 pairs/4 pairs

$217.8^{\circ} \mathrm{C}$

Stainless steel tubes

Carbon steel shells

Vertical canned centrifugal

3

555

360

Horizontal centrifugal

2

830

810

Steam-turbine 


\section{B. 3 SUPERHEAT CYCLE}

Figure B-2 shows the schematic diagram of the superheat cycle. This steam cycle is similar to that considered in Gas-Cooled Fast Reactor designs. Steam conditions at the turbine inlet are $9.24 \mathrm{MPa}$ and $534^{\circ} \mathrm{C}$ (1340 psia and $994^{\circ} \mathrm{F}$ ). The turbine-generator selected is a tandem compound 1,800 rpm unit operating with a superheat cycle, and it consists of one high pressure (HP) cylinder, one intermediate pressure (IP) cylinder, two low pressure (LP) cylinders, and one generator. This cycle does not use a moisture separator-reheater.

Figure B-4 shows the schematic heat and mass balance diagram of this conversion system. The condensate system, feedwater heating system, and heat rejection system are the same as described in Section B.2 of this appendix. 


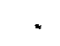

. 


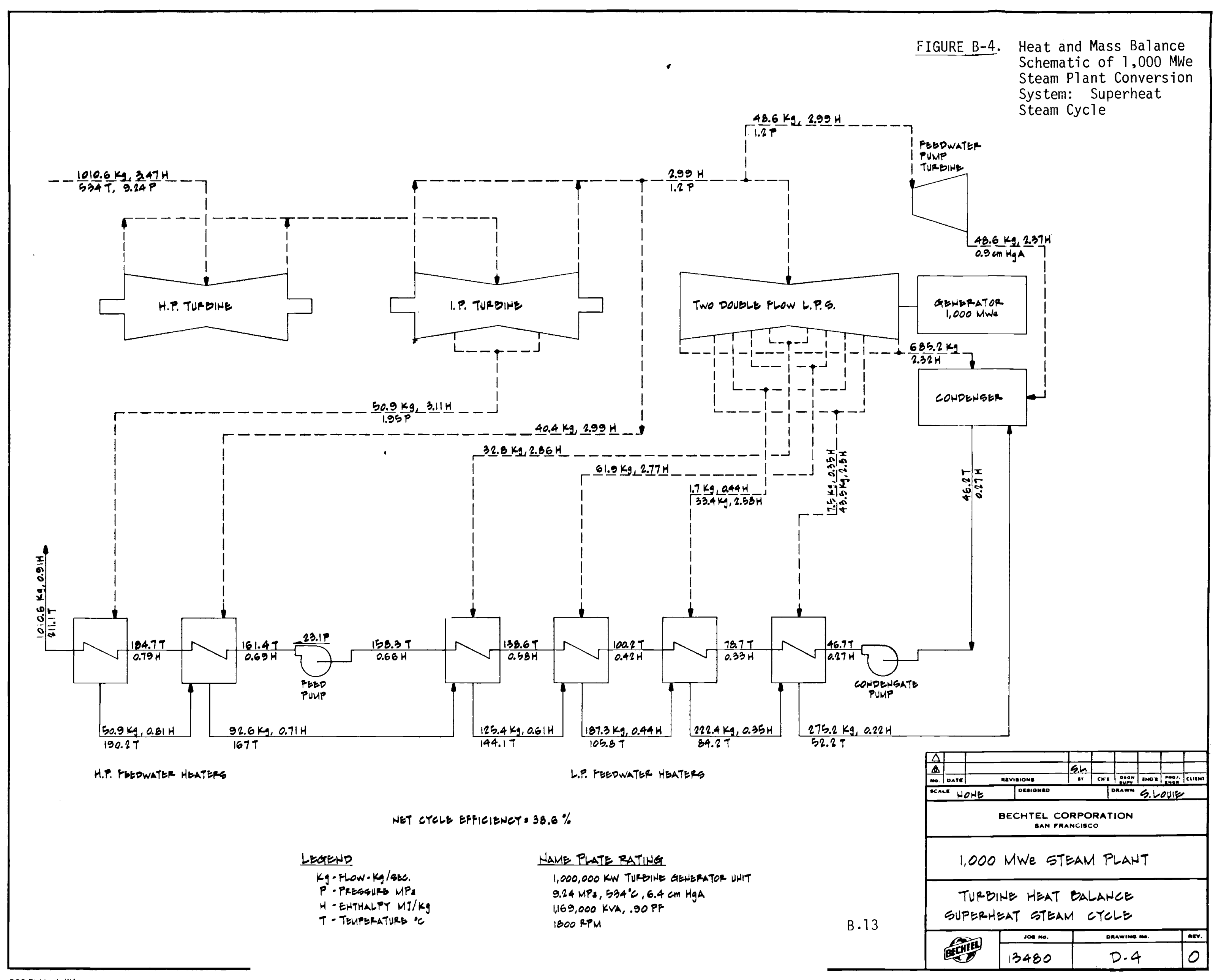



. 


\section{EQUIPMENT LIST - FIGURE B-4}

Major equipment along with their capacity ratings for the superheat cycle are listed below:

Main Turbine Data

Rating, $\mathrm{kW}$

Speed, rpm

Throttle steam pressure, $\mathrm{MPa}$

Throttle steam temperature, ${ }^{\circ} \mathrm{C}$

Steam flow, $\mathrm{kg} / \mathrm{sec}$

Condenser pressure, $\mathrm{cm} \mathrm{Hg}$. A
$1,000,000$

1,800

9.24

534

1010.6

6.4

Generator

Type

Rating, kVA/kV

Generator pf

Hydrogen pressure, $\mathrm{MPa}$

Cooling Tower

Type

Flow rate, $\mathrm{kg} / \mathrm{sec}$

No. of cel1s (tota1)

Approach, ${ }^{\circ} \mathrm{C}$

Range, ${ }^{\circ} \mathrm{C}$

No. of Fans

Turbine Condenser

Quantity

Type

Surface area: $\mathrm{m}^{2}$

Tube material

Duty, MWt

Cooling Water Flow, $\mathrm{kg} / \mathrm{sec}$

Circulating Water Pump

Quantity

Type

Capacity, kg/sec

Head, m

Driver, kW
4 pole, 3 phase, $60 \mathrm{~Hz}$

$1,169,000 / 22$

0.9

0.52
Induced draft

21,500

24 (12 cells per tower)

4.4

17.3

24
1

Dual pressure - 2 she11s

57,728

Admiralty Brass

1558

21,500
3

Vertical wet pit 7,200

26

Electric motor, 2,600 


\section{EQUIPMENT LIST (contd)}

Condensate Pump

Quantity

Type

Capacity, kg/sec

Head, m

3

Vertical

340

380

Feedwater Pumps

Quantity

Type

Design Capacity, $\mathrm{kg} / \mathrm{sec}$

Head, $m$

2

Horizontal barrel

340

2450

Feed Pump Turbine

Quantity

Type

Rating, kW

Steam Flow, $\mathrm{kg} / \mathrm{sec}$

Inlet conditions, $\mathrm{MPa} / \mathrm{MJ} / \mathrm{kg}$

2

Multistage, variable speed

17,500

48.6

$1.2 / 2.99$

Feedwater heater

Type

"U" Tube

Number, HP/LP

2 pairs/4 pairs

Final Feedwater Temperature

$211^{\circ} \mathrm{C}$

Material

$3045 s$ tubes

Carbon steel shells 


\section{B.4 PLANT ARRANGEMENT}

Turbine Building Arrangement

A general arrangement of the steam turbine building was developed for each of the saturated and superheat steam cycle cases. In each case, a plan view and a cross-sectional view of the turbine building was prepared. Drawings $B-5$ and B-6 are respectively the plan and cross-sectional views of the saturated cycle case. Drawings B-7 and B-8 are the respective plan and cross-sectional views of the superheat cycle case. These drawings show the arrangement of the major equipment and piping in the turbine building. The major equipment includes the turbine-generator, moisture separator-reheater, condenser, feedwater heater, and feed pump. It is to be noted that the superheat cycle does not require a moisture separator-reheater as may be seen in the heat balance diagram (Figure B-4) and the arrangement drawings (Drawings B-7 and B-8) for this cycle.

Plot Plan

In addition to the turbine building arrangement drawings, a plot plan was prepared (Drawing B-9) showing the relationship between the turbine building, the cooling towers, and other facilities associated with the steam plant. This plot plan is intended to show the relative location of the various structures and facilities of a power plant. It is to be noted that the plot plan does not show the reactor building, heat exchanger building, and other related buildings as these were not addressed in this appendix (Appendix $B$ ). However, a general area is indicated for these buildings in the plot plan. 



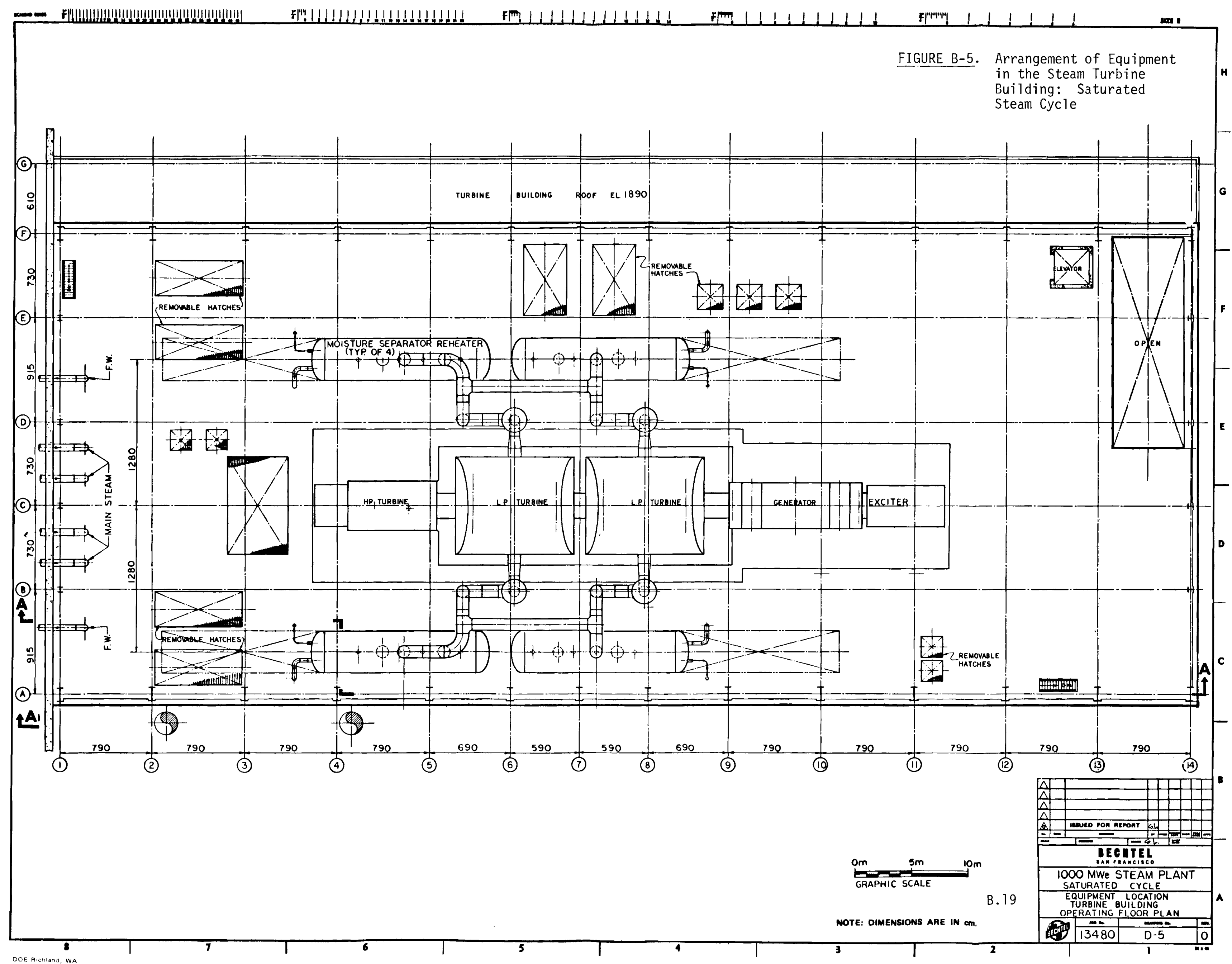




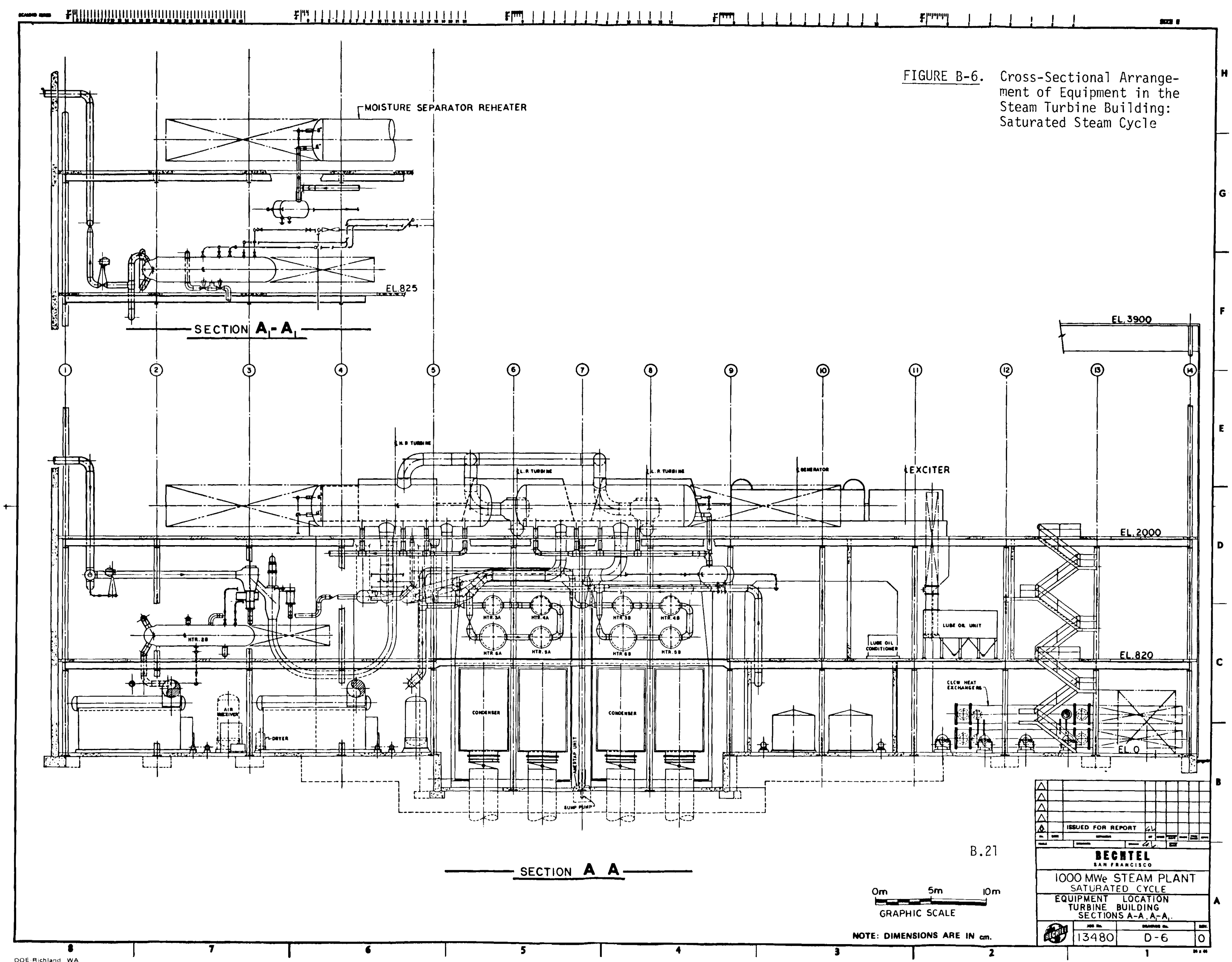




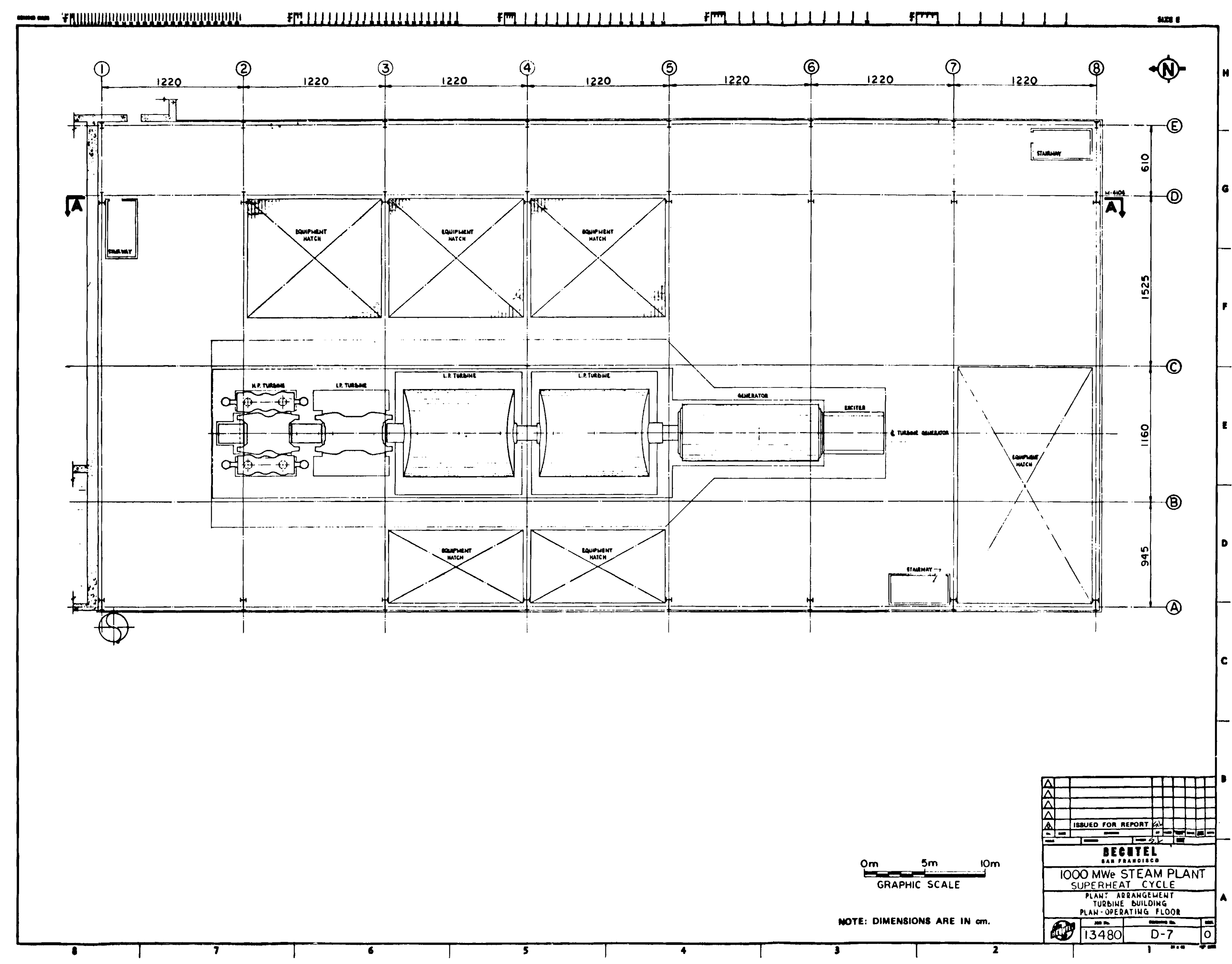

FIGURE B-7. Arrangement of Equipment in the Steam Turbine Building: Superheat Stean 


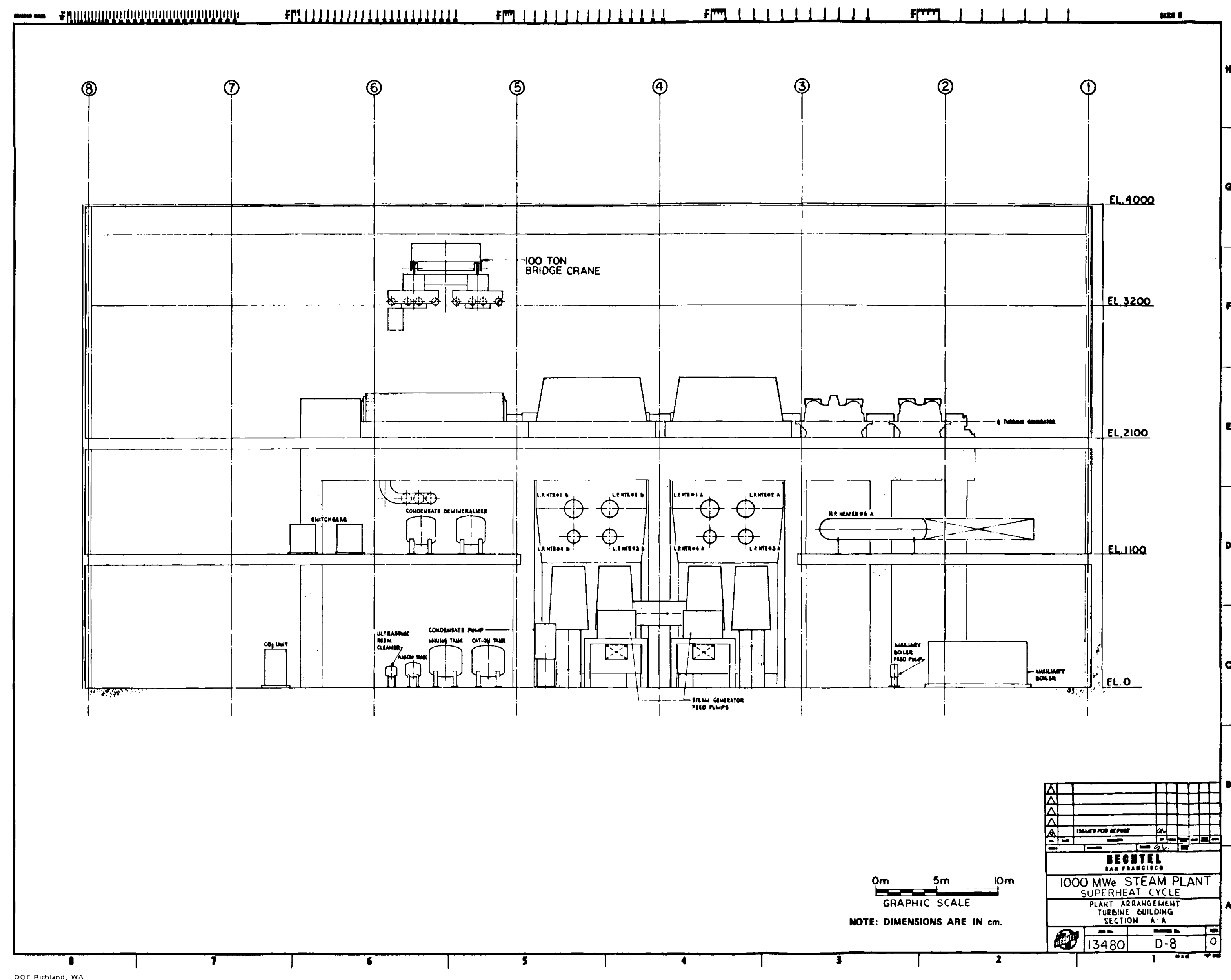

FIGURE B-8. Cross-Sectional Arrangement of Equipment in the steam Turbine Building:

Superheat Steam Cycle

1

3

2 
. 


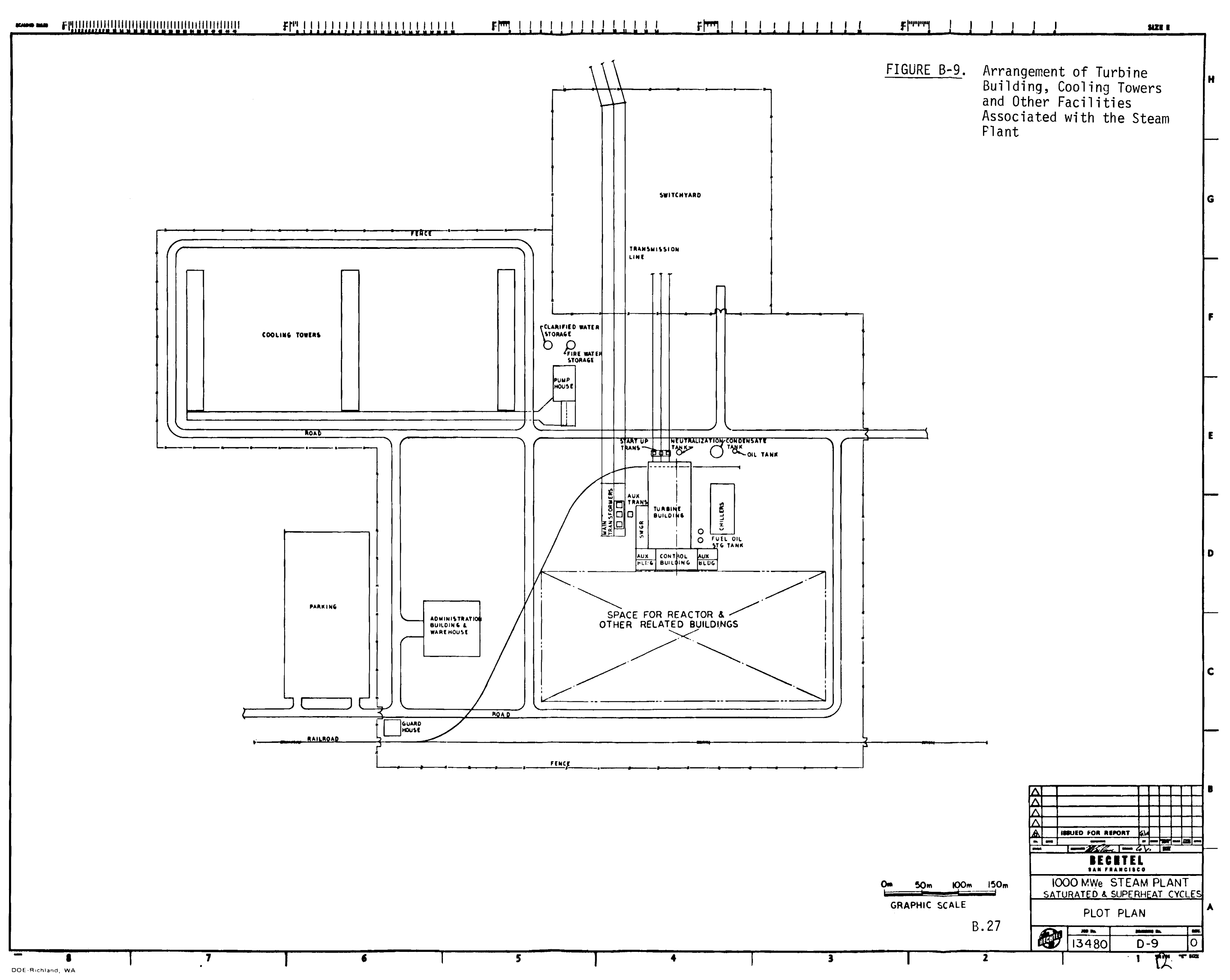




$$
\text { . }
$$


APPENDIX C

REFERENCE POWER CONVERSION PLANT COST ESTIMATE 


\section{REFERENCE POWER CONVERSION PLANT COST ESTIMATE}

[The estimates and scaling rules presented herein are based largely on adaptations and extrapolations of previous design studies of fission and fusion plants performed by Bechtel, particularly the 1000 MWe (gross) Prototype Large Breeder Reactor (PLBR) study developed by General Electric Company and Bechtel Corporation. The estimate for the PLBR plant was based on general arrangement drawings, electrical single-line diagrams, system flow diagrams, and a plot plan for a hypothetical site. Materials and equipment were prices at January 1976 cost levels, with manual labor at $\$ 13.75$ per hour, representing an appropriate mix of construction crafts. Adequate skilled labor was assumed to be available to support a straight time single shift work week. More recent PLBR cost studies provided information comparing saturated and superheated steam cycles. The PLBR estimates and cost studies were escalated to 1979 dollar levels, and allocated to the appropriate fusion plant cost accounts from PNL-2648. Caution should be exercised when applying these results. The system being scaled should represent systems similar in design, scope and interface to the reference designs (Appendix $B$ ); the scaling rules of Appendix $D$ should be applied within the applicable ranges as indicated for the rules. The application of these results should be limited to conceptual design studies]. 
CAPITAL COST DETAIL

(1979 Dollars)

Accounts

\$Million

21

STRUCTURES \& SITE FACILITIES

.03 Turbine and Control Buildings

.01 Basic Building Structures

16.2

.02 Building Services

$\underline{9.2}$

.04 Cooling System Structures

4.2

23

TURBINE PLANT EQUIPMENT

.01 Turbine Generators

134.9

.01 Turbine Generator \& Accessories

62.5

.02 Foundations

60.6

1.9

.02 Main Steam System (Piping)

4.8

.03 Heat Rejection Systems

18.5

.01 Water Intake Common Facilities

1.0

.02 Circulating Water Systems

5.5

.03 Cooling Towers

$\underline{12.0}$

.04 Condensing System

13.1

.01 Condensers

9.1

.02 Condensate System

3.5

.03 Gas Removal Systems

$\underline{0.5}$

.05 Feed Heating System

.01 Regenerators \& Recuperators

9.5

.02 Pumps

3.5

.03 Tanks

5.9

0.1

.06 Other Turbine Plant Equipment

10.0

.01 Turbine Auxiliaries

0.4

.02 Auxiliaries Cooling System

3.4

C.2 


\section{SATURATED STEAM CYCLE (contd)}

CAPITAL COST DETAIL

(1979 Dollars)

Accounts

\$Million

.06 Other Turbine Plant Equipment (contd)

10.0

.03 Make-up Treatment System

2.2

.04 Chemical Treatment System

3.4

.05 Central Lubrication System

$\underline{0.6}$

.07 Instrumentation \& Control

16.5

ELECTRIC PLANT EQUIPMENT

.01 Switchgear

117.6

.01 Generator Circuits

8.7

.02 Station Service

1.3

7.4

.02 Station Service Equipment

11.4

.01 Station Service \& Start-up Transformer

1.6

.02 Low Voltage Substations \& Transformer

2.4

.04 Diesel Engine Generators

2.8

.07 Main Power Transformers

4.6

.03 Switchboards

0.1

.04 Protective Equipment

1.3

.05 Electrical Structures \& Wiring Containers

52.1

.01 Concrete Cable Tunnels \& Envelopes

1.8

.02 Cable Trays \& Support

12.7

.03 Conduit

$\underline{37.6}$

.06 Power \& Control Wiring (including heat tracing)

.01 Generator Circuits Wiring

.02 Station Service Power Wiring

.03 Control Wiring 
SATURATED STEAM CYCLE (contd)

CAPITAL COST DETAIL (1979 Dollars)

Accounts

\begin{tabular}{ccc} 
& \$Million & \\
& 2.1 & 15.5 \\
& & \\
& 3.3 & \\
& & \\
1.0 & & \\
8.4 & \\
0.9 & & \\
0.7 & \\
0.1 & \\
1.1 & & \\
\hline
\end{tabular}

.07 Electric Lighting

25 MISCELLANEOUS PLANT EQUIPMENT

.01 Transportation \& Lifting Equipment

.01 Cranes, Hoists, Monorails, Conveyors

.02 Air \& Water Service Systems

.01 Air Systems

.02 Water Systems

.03 Communications Equipment

.01 Local Communication Systems

.02 Signal Systems

.04 Furnishing \& Fixtures

.01 Safety Equipment

.02 Shop, Laboratory \& Test Equipment

1.1 


\section{SUPERHEAT STEAM CYCLE \\ i \\ CAPITAL COST DETAIL \\ (1979 Dotlars)}

Accounts

\$illion

21

STRUCTURES \& SITE FACILITIES

30.6

.03 Turbine and Control Buildings

26.4

.01 Basic Building Structures

17.2

.02 Building Services

9.2

.04 Cooling System Structures

4.2

23

TURBINE PLANT EQUIPMENT

140.7

.01 Turbine Generators

66.4

.01 Turbine Generator \& Accessories

64.5

.02 Foundations

1.9

.02 Main Steam System (Piping)

5.9

.03 Heat Rejection Systems

11.9

.01 Water Intake Common Facilities

0.6

.02 Circulating Water Systems

3.5

.03 Cooling Towers

7.8

.04 Condensing System

.01 Condensers

13.1

.02 Condensate System

9.1

.03 Gas Removal Systems

3.5

$\underline{0.5}$

.05 Feed Heating System

.01 Regenerators \& Recuperators

6.2

.02 Pumps

10.5

.03 Tanks

0.2

.06 Other Turbine Plant Equipment

10.0

.01 Turbine Auxiliaries

0.4

.02 Auxiliaries Cooling System

3.4

.03 Make-up Treatment System

2.2

C.5 
CAPITAL COST DETAIL (1979 Dollars)

Accounts

.06 Other Turbine Plant Equipment (contd) .04 Chemical Treatment System .05 Central Lubrication System

.07 Instrumentation \& Control

24 ELECTRIC PLANT EQUIPMENT

.01 Switchgear

.01 Generator Circuits

.02 Station Service

.02 Station Service Equipment

.01 Station Service \& Start-up Transformer

.02 Low Voltage Substations \& Trans former

.04 Diese1 Engine Generators

.07 Main Power Transformers

.03 Switchboards

.04 Protective Equipment

.05 Electrical Structures \& Wiring Containers .01 Concrete Cable Tunnels \& Envelopes

.02 Cable Trays \& Support

.03 Conduit

$.06 \frac{\text { Power \& Control Wiring (including heat }}{\text { tracing) }}$

.01 Generator Circuits Wiring

.02 Station Service Power Wiring

.03 Control Wiring

7.4 29.7

\$Million

10.0

3.4

0.6

16.5

122

8.7

1.3

11.4

1.6

2.4

2.8

4.6

0.1

1.3

54.4

1.8

13.3

39.3

44.2

11.5 
CAPITAL COST DETAIL

(1979 Do11ars)

Accounts

\begin{tabular}{ccc} 
& \$Million & \\
& 2.1 & \\
& & 15.5 \\
& 3.3 & \\
\hline .3 & & \\
1.0 & & \\
8.4 & & \\
0.9 & & \\
0.7 & & \\
0.1 & & \\
1.1 &
\end{tabular}





\section{APPENDIX D}

\section{REFERENCE POWER CONVERSION PLANT COST ESTIMATE}

SCALING RULES 


\section{SCALING RULES - REFERENCE POWER CONVERSION PLANT COST ESTIMATE}

[Caution should be exercised when applying these rules. The system being scaled should represent systems similar in design, scope and interface to the reference designs (Appendix B); scaling rules should be applied within the applicable ranges as indicated for the rules. The application of these results should be limited to conceptual design studies. For further qualifications, see Appendix C].

The data presented in this appendix provide means for adjusting the Reference Power Conversion Plant Cost Estimate (Appendix C) to reflect different design conditions than those outlined in the Reference Power Conversion Plant Description (Appendix $B$ ). For each account, cost/design relationships are discussed together with scaling factors that apply to the costs in that account. These cost relationships are applicable to the total cost of the account within a 1 imited range of deviations from the reference plant design if noted in the discussion of that account, and in any case within an overall plant range from 800 MWe to 1400 MWe (gross). They are not appropriate for preparing original estimates of substantially different design.

21.03 Turbine Building (including Control Room, Heater Bay, Diesel Generator and Auxiliary Equipment Areas)

This account may be adjusted by applying the appropriate building volume unit cost for each of the major building functions: for the Turbine Building area, $\$ 83 / \mathrm{m}^{3}$ of enclosed space; for the Control Room area, $\$ 197 / \mathrm{m}^{3}$; for the Diesel Generator and Auxiliary Equipment areas, $\$ 777 / \mathrm{m}^{3}$. These unit costs apply to differences in volume from the reference design case which is based on volumes for these functional areas as follows: Turbine Building $182,500 \mathrm{~m}^{3}$, Control Room $7,400 \mathrm{~m}^{3}$ and Diesel Generator and Auxiliary Building areas, $49,000 \mathrm{~m}^{3}$. These building volumes are consistent with recent nuclear plant designs which require approximately $200 \mathrm{~m}^{3} /$ MWe to $250 \mathrm{~m}^{3}$ /Mwe for these types of buildings. It is expected that fusion plants will have similar requirements. 


\subsection{Cooling System Structures}

The reference design employs a closed condenser cooling system, thus no intake, discharge or unpressurized conduit systems are included. Recirculating structures (Account 21.04.04) and Cooling Tower System Earthwork (Account 21.04.05) may be adjusted by the following relationship:

$$
c_{1}=C_{R}\left[\frac{M W e_{1}}{\overline{M W e}_{R}}\right]^{\cdot 3}
$$

where $C_{1}=$ adjusted cost

$C_{R}=$ reference cost

$M \mathrm{~N}_{\mathrm{R}}=$ rating of reference turbine equipment (Appendix $B$ )

$\mathrm{MWe}_{1}=$ rating of new turbine equipment

\subsection{Turbine-Generator}

The turbine-g Generator account includes the purchase and installation of the turbine-generator and associated auxiliary equipment and piping as well as the turbine pedestal. The account may be adjusted in relation to the gross generating capacity of the equipment by means of the following relationship:

$$
C_{1}=C_{R}\left[\frac{\mathrm{MWe}_{1}}{\mathrm{MWE}_{\mathrm{R}}}\right]^{\cdot 7}
$$

This relationship is applicable in the case of saturated steam conditions within the temperature range of $500^{\circ} \mathrm{F}$ to $750^{\circ} \mathrm{F}$, and within the pressure range of $900 \mathrm{psi}$ to $1200 \mathrm{psi}$. The same relationship is applicable in the case of superheated steam conditions within the temparature rarige of $800^{\circ} \mathrm{F}$ to $1000^{\circ} \mathrm{F}$, and within the pressure range of 1800 psi to $2400 \mathrm{psi}$.

\subsection{Main Steam System (Piping)}

The main steam system cost is largely affected by the steam flow through the turbine generator as well as the length of steam piping as determined from the plant arrangement. Alterations from the reference facility can be calculated from the expression:

$$
C_{1}=C_{R}\left[\frac{M W t_{1}}{M W t_{R}} \times \frac{\text { LF Main Steam Piping }}{6,400 \mathrm{LF}}\right]
$$


where: $\quad L F=$ total 1 inear feet of piping, including expansion loops

$M W t_{R}=$ thermal rating of the reference $p l a n t$ (Appendix $B$ )

$\mathrm{MWt}_{1}=$ thermal rating of the new plant

23.03 Heat Rejection Systems

The heat rejection system, composed of the facilities associated with the circulating water pumps and motor drives, and the waste heat cooling tower, is sensitive in cost mainly to the amount of heat rejected. Given a constant plant efficiency, adjustment of the cost from the reference plant can be determined from the expression:

$$
c_{1}=c_{R}\left[\frac{M W t_{1}}{M W t_{R}}\right]^{.8}
$$

\subsection{Condensing System}

The steam condensing system cost is affected primarily by the size of the condenser, and is related to the steam flow through the turbine and the amount of heat rejected. A direct relationship of cost per MWe gross generator output is sufficiently accurate for adjusting condensing system cost:

$$
c_{1}=c_{R}\left[\frac{M W e_{1}}{M_{R}}\right]^{\cdot 9}
$$

\subsection{Feedwater Heating System}

The feedwater heating system, composed of pumps, heat exchangers, piping, valves, and miscellaneous equipment is sensitive in cost mainly to the feedwater flow and temperature rise. The cost relationship to the flow, however, is less than linear, and the expression

$$
c_{1}=c_{R}\left[\frac{M W t_{1}}{M W t_{R}}\right]^{\cdot 6}
$$

may be used to adjust this account for differences in gross plant heat transport capacity.

\subsection{Other Turbine Plant Equipment}

This account may be related to plant capacity by the expression: 


$$
c_{1}=c_{R}\left[\frac{M \mathrm{We}_{1}}{M \mathrm{Me}_{\mathrm{R}}}\right]^{\cdot 6}
$$

\subsection{Instrumentation and Control Equipment}

This account includes instrumentation and control equipment for the turbine plant balance of plant facilities, and undergoes modest change in cost within the range of plant sizes between 800 MWe and 1400 MWe (net). Its cost may be estimated from the reference plant cost by the expression:

$$
c_{1}=C_{R}\left[\frac{M e_{1}}{M W e_{R}}\right]^{\cdot 3}
$$

\section{Electric Plant Equipment}

The electric plant accounts consist mainiy of equipment and materials required to distribute power to the in-plant loads associated with the reactor plant, the turbine plant and other balance of plant equipment. Therefore, the total auxiliary load required to operate equipment throughout the entire plant is one important parameter of electric plant cost, the other being building sizes, which affects the average run lengths and quantities of wire, cable, conduit, etc.

The electric plant accounts provide for equipment and feeder cabling to to the reacotr power supply area, but do not include reactor power supply equipment, conductros, switches or protective devices beyond the first transformer feeding the reactor power supply equipment.

24.01, 24.02, 24.03. Major electrical equipment accounts may be assumed to vary in cost, in direct relationship to the auxiliary loads required by the plant mechanical equipment. Thus, adjustments to those accounts may be made by use of the expression:

$$
c_{1}=C_{R}\left[\frac{\text { MWaux }_{1}}{\operatorname{MWaux}_{R}}\right]
$$

where MWaux 1 and MWaux $R$ are plant auxiliary loads (exclusive of power loads required by the reactor power supply equipment) for the adjusted and reference plants, respectively. 
24.04 Protective Equipment (General Station Grounding Systems and Cathodic Protection)

The cost of grounding and cathodic protection systems is composed mainly of the station grounding grid and ground connecting cables. Adjustments to the cost of this account may be related to the plot area requirements for all buildings by the expression:

$$
c_{1}=c_{R}\left[\frac{A_{1}}{A_{R}}\right]
$$

where $A_{1}$ and $A_{R}$ are the total plot area requirements for all buildings in the new and reference plants, respectively, and $C_{1}$ is held within the range of $10 \%$ less than $C_{R}$, to $200 \%$ greater than $C_{R}$. For the reference case, the $A_{R}$ is $14,000 \mathrm{~m}^{2}$.

24.05 and 24.06. The estimated costs for these accounts are influenced by the plant auxiliary load requirement, which impacts numbers and sizes of cabling runs; and building sizes, which impacts average run lengths. Adjustments to cost may be estimated with equal weight to each of these parameters by means of the expression:

$$
C_{1}=0.5 C_{R}\left[\frac{\text { MWaux }_{1}}{\text { MWaux }_{2}}+\frac{A_{1}}{A_{R}}\right]
$$

The estimated costs in Accounts 24.05 and 24.06 include allowance for electric heat tracing of primary and secondary liquid metal heat transport system piping. For gas cooled reactor designs where tracing of heat transport 1 ines is not required, $\$ 2$ million should be deducted from Account 24.05 and $\$ 4$ million from Account 24.06 before making the adjustments described above. Adjustments to Accounts 24.05 and 24.06 by means of the expression above should be limited to values of $C_{7}$ which are within the range of $20 \%$ less than $C_{R}$ to $100 \%$ greater than $C_{R}$. If the formula indicates values beyond this range, independent assessment of the value is necessary. 
24.07 Electric lighting may be adjusted in direct proportion to total volume of all buildings:

$$
c_{1}=\left[\begin{array}{ll}
c_{R} & V_{1} \\
V_{R}
\end{array}\right]
$$

where $V_{1}$ and $V_{R}$ are total volumes of al1 buildings (in accounts 21.02, 21.03, 21.05 and 21.06) for the adjusted and reference plants, respectively, $V_{R}$ being $700,000 \mathrm{~m}^{3}$.

25. Miscellaneous Plant Equipment

Miscellaneous plant equipment may be left unadjusted, except for Account, 25.01, Transportation and Lifting Equipment. With this account, $\$ 800,000$ represents an allowance for purchase and installation of the reactor building crane. For special fusion reactor configurations, special reactor building cranes are to be estimated independently and substituted for this $\$ 800,000$ allowance. 
APPENDIX E

COST ESTIMATING RULES

Spare Parts Allowance/

Contingency Allowance/

Design Allowance 


\section{COST ESTIMATING RULES}

Design allowances, contingency allowances and spare parts allowances are dollar amounts added to estimates to account for costs expected to be incurred during the course of a project, but which are not adequately accounted for in the body of the estimate.

DESIGN ALLOWANCE

A design allowance is usually employed in an estimate of a particular piece of equipment or system for which a quotation was received or an estimate was made at an early stage of the design of that item. The allowance represents the difference between the simpler concept envisioned at the time the estimate was made and the mature design expected to be developed ultimately. It may be considered as equivalent to change orders to a supplier if a purchase order was written when the estimate was made.

For a fusion plant estimate, accounts $21,23,24$, and 25 represent facilities and systems that are similar to such facilities and systems in a conventional fission plant and which are well understood in concept. Therefore, no design allowance for these accounts is recommended.

However, for a fusion plant estimate, account 22 represents equipment and systems that are not conventional in design. Therefore, a design allowance of $10 \%$ of estimated costs is recommended.

\section{CONTINGENCY ALLOWANCE}

The contingency allowance is an amount added to an estimate to account for the difference between the sum of individual items estimated and the total amount that is reasonabiy expected to be spent, considering the degree of uncertainties in the estimated quantities, prices, labor productivity, etc. embodied in the estimate details. Due to the nature of estimating, which is generally to assign values to known quantities, unknown quantities and other uncertainties usually tend to produce a net increase from the estimated costs. Thus, with all estimates there is the risk that in the course of design and construction, actual costs will be greater than the estimate. The contingency allowance is added to an estimate to reduce the risk of overrun to an acceptable level. The magnitude of the allowance must be considered based on a thorough understanding of the methods and bases of the estimated costs. 
For the conceptual level of design for fusion plant accounts $21,22,23$, 24 , and 25 , a contingency al lowance of $15 \%$ of estimated costs is recommended. SPARE PARTS ALLOWANCE

Although the types and numbers of spare parts to be provided in inventory at the startup of a power plant is decided by the owner of the plant, common practice indicates that a reasonable cost allowance for the value of spares in inventory falls in a fairly consistent range of percentages of major equipment costs. This allowance may also be expressed as a function of total cost of installed equipment and materials, since the relationship between major equipment cost and total cost of equipment and materials remains relatively constant.

Spare parts allowances of $2 \%$ of estimated costs for account $21,2 \%$ of estimated costs for account $22,2 \%$ of estimated costs for account $23,4 \%$ of estimated costs for account 24 , and $3 \%$ of estimated costs for account 25 are recommended. 


\section{APPENDIX $F$}

STANDARD UNIT COSTS

Operating and Maintenance/

Scheduled Component Replacement 
STANDARD UNIT COSTS - Operation and Maintenance

Management Personne1

Operations Personne1

Maintenance (Routine) Personnel

Maintenance (Special) Personnel

Technical Personnel

Clerical and Services Personnel

\begin{tabular}{|c|c|}
\hline 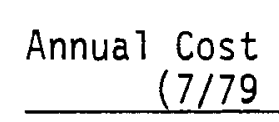 & $\begin{array}{l}\text { Per Staff Member (a } \\
\text { Price Levels) }\end{array}$ \\
\hline & $\$ 75,000$ \\
\hline & $\$ 55,000$ \\
\hline & $\$ 40,000$ \\
\hline & $\$ 55,000$ \\
\hline & $\$ 40,000$ \\
\hline & $\$ 30,000$ \\
\hline
\end{tabular}

\begin{tabular}{l} 
Annual Cost Per Staff Member(a) \\
$(7 / 79$ Price Levels $)$ \\
\hline
\end{tabular}

$\$ 75,000$

$\$ 40,000$

$\$ 30,000$

(a) Includes fringe benefits, insurance, and overhead expense burdens. 


\section{APPENDIX G}

STANDARD UNIT COSTS

Fuel/Special Materials 
STANDARD UNIT COSTS - FUEL/SPECIAL MATERIALS

Purchase Cost (price)

(7/79 Price Levels)

02.-03 FUEL

26. SPECIAL MATERIALS

26.01 to 26.04 Fuel and Coolants

Deuterium ( $99.75 \%$ pure)

$$
\mathrm{D}_{2} \mathrm{O}
$$

$\$ 225 / \mathrm{kg}$

$\mathrm{D}_{2}$

$\$ 2000 / \mathrm{kg}$

Tritium (current market price)

$\$ 5000 / 9$

Tritium (mature fusion ecomomy)

$\$ \quad 0 / 9$

Lithium (reactor grade)

$\$ 30 / \mathrm{kg}$

Helium

Sma11 shipments

$\$ 3.25 / 1$ iter

Bulk quantities

$\$ 2.00 / 1$ iter

G.1 


\section{DISTR IBUT ION}

No. of

Copies

\section{OFFSITE}

\author{
A A. Churm \\ DOE Chicago Patent Group \\ 9800 S. Cass Avenue \\ Argonne, IL 60439 \\ C. Head \\ DOE Office of Fusion Energy \\ Washington, DC 20545
}

J. N. Grace

DOE Office of Fusion Energy

Washington, DC 20545

J. Baublitz

DOE Office of Fusion Energy Wash ington, DC 20545

F. E. Coffman

DOE Office of Fusion Energy Wash ington, DC 20545

J. F. Decker

DOE Office of Fusion Energy Wash ington, DC 20545

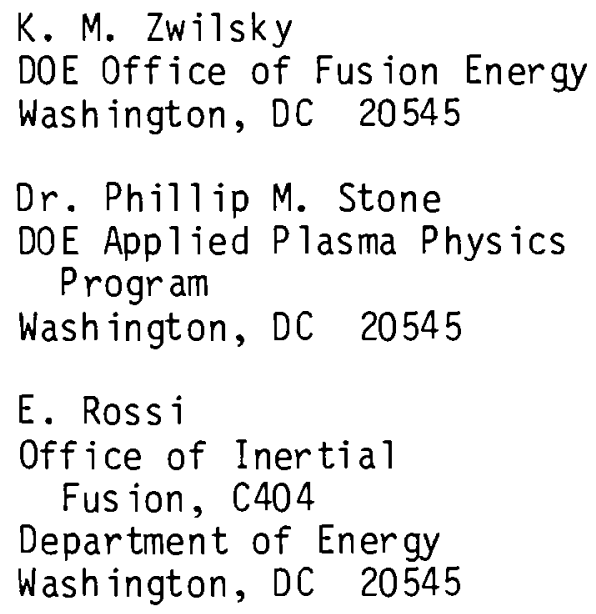

No. of

Copies

\section{H. M. Busey}

DOE Division of Military Application Washington, DC 20545

M. A. Bell

DOE Division of Safety Standards and Compliance Wash ington, DC 20545

117 DOE Technical Information Center

W. E. Parkins, Manager Atomics International Component Engineering and Technology Division North Amer ican Rockwell

P.0. Box 309

Canoga Park, CA 91304

Mark Triplett

Battelle Columbus Laboratories

$505 \mathrm{King}$ Avenue

Columbus, $\mathrm{OH} 43201$

R. Aronstein Bechte 1 Corporation P.0. Box 3965

San Francisco, CA 94119

S. Ghose Bechte 1 Corporation P. 0. Box 3965 San Francisco, CA 94119

A. J. Impink, Jr. Carnegie Melion University Pittsburgh, PA 15213 
No. of

Copies

D. H. Berwald

Exxon Research and Engineering Company

P.0. Box 8

Linden, NJ 07036

G. R. Hopk ins

Gulf General Atomic

P.0. Box 1111

San Diego, CA 92112

Zeinab Sabri

Iowa State University

261 Sweeney Hall

Nuclear Engineering Dept.

Ames, IA 50010

W. Bauer

Division Supervisor of

Physical Research

Sandia Labs Livermore

Livermore, CA 94550

Rolf Buenoe

Max-Planck-Institut für

Plasmaphys ik

8046 Garching Bei München

WEST GERMANY

J. J. Reinmann

NASA-Lew is Research Center

2100 Bookpark Road

Clevel and, $\mathrm{OH} \quad 44135$

Vincent Arp

National Bureau of Standards

Cryogenics Division

Boulder, CO 80302

R. A. Huse

Public Service Electric and Gas Company

80 Park Place

Newark, NJ 07101
No. of

Copies

J. Mitchiner

Sandia Laboratories

P.0. Box 5800

Albuquerque, NM 87115

M. Kristiansen

Texas Tech. University

Lubbock, TX 79409

A. F. Haught

United Aircraft Research Laboratory

United Aircraft Corporation

East Hartford, CT 06108

Dean Abrahamson

University of Minnesota

School of Public Affairs

Social Science Building/309

Minneapolis, MN 55455

Abraham Hertzberg

University of Washington

Aerospace Research Laboratory

316 Guggenheim

Seattle, WA 98105

A. L. Babb

University of Washington

Nuclear Engineering Dept.

Seattle, WA 98105

E. E. Donaldson

Washington State University

Department of Physics

Pullman, WA 99163

Les 1 ie S. Ramsey

450 North 5 th Street

Indianna, PA 15701

E. Beckner

Sandia Laboratories

P.0. Box 5800

Albuquerque, NM 87115 
No. of

Copies

J. Clayton

KMS Fusion, Inc.

P.0. Box 1567

Ann Arbor, MI 48106

ONSITE

2 DOE Richland Operations Office

W. A. Burns

H. E. Ransom

2 Hanford Engineering Development Laboratory

D. G. Doran

H. H. Yoshikawa
No. of

Copies

60 Pacific Northwest Laboratory

W. E. Bickford

D. E. Deonigi (2)

D. A. Dingee (2)

H. R. Gardner

R. H. Jones

B. R. Leonard

A. D. Rockwood

S. C. Schulte (40)

V. L. Teofilo

C. E. Willingham

T. L. Willke (2)

Technical Information (5)

Publishing Coordination RO(2) 

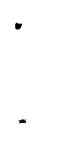